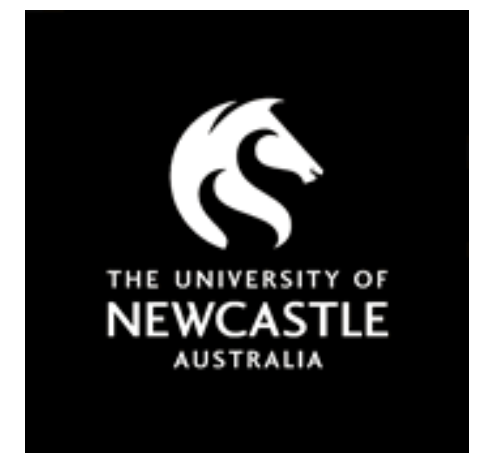

NOVA

University of Newcastle Research Online

nova.newcastle.edu.au

Bournival, Ghislain; Ata, Seher; Wanless, Erica J. "Behavior of bubble interfaces stabilized by particles of different densities", Published in Langmuir Vol. 32, Issue 25, p. 6226-6238. (2016)

Available from: $\underline{\text { http://dx.doi.org/10.1021/acs.langmuir.6b00656 }}$

This document is the Accepted Manuscript version of a Published Work that appeared in final form in Langmuir, copyright (c) American Chemical Society after peer review and technical editing by the publisher. To access the final edited and published work see http://dx.doi.org/10.1021/acs.langmuir.6b00656.

Accessed from: http://hdl.handle.net/1959.13/1323784 


\title{
The behavior of bubble interfaces stabilized by particles of different densities
}

Ghislain Bournival $^{1}$, Seher Ata ${ }^{1,{ }^{*}}$ and Erica J. Wanless ${ }^{2}$

\begin{abstract}
${ }^{1}$ The School of Mining Engineering, The University of New South Wales, Sydney, NSW, 2052, Australia.

${ }^{2}$ Priority Research Centre for Advanced Particle Processing and Transport, The University of Newcastle, Callaghan, NSW, 2308, Australia.
\end{abstract}

${ }^{*}$ To whom correspondence should be addressed

Email: s.ata@unsw.edu.au 


\begin{abstract}
The stability of bubbles laden with particles of different densities has been investigated. Capillary-held bubbles were produced and coated with particles across the density range $1.2-3.6 \mathrm{~g} \cdot \mathrm{cm}^{-3}$. The materials used were poly(methyl methacrylate) (PMMA), glass, and anatase. The interaction of the bubbles, once brought into contact, was monitored using high-speed video recording. Visual inspection indicated that denser particles were more easily displaced during the contact of the bubbles, and therefore that the PMMA particles provided a particle barrier more resistant to coalescence. The coalescence events yielded information on the surface properties of the bubble and the detachment of particles. Attached particles commonly dampen the oscillation of the coalesced bubbles through viscous drag and change in surface properties (e.g. area exclusion principle). The dampening of the oscillation generally leads to a reduced mass of particle detaching from the bubble surface. It was found that the different materials investigated did not offer clear evidence on the effect of particle detachment on the bubble surface properties in the present systems. On the other hand, the detachment of different particle materials seemed to be consistent with one another when comparing the attachment and detachment forces exerted on the particles based on their density, size, and hydrophobicity. It was concluded that particles of lower density are more effective at stabilising interfaces and thus particle density is an important parameter in the selection of materials for the handling of dispersions.
\end{abstract}

\title{
Keywords
}

Bubble stability, coalescence, particle, density, detachment

\section{Introduction}

The interaction of solid particles with interfaces, whether liquid-gas or liquid-liquid, is commonplace in many processes. Emulsion droplets and foam bubbles may be stabilised by micron- nano-sized particles, which are smaller than the bubble (or droplet), and where the particles are not perfectly wetted by either phase. In all cases, there are generally three primary processes for the attachment of particles onto an interface ${ }^{1}$ (i) the approach of the interface (e.g. emulsion droplet or air bubble) to the particle thus forming a thin liquid film, (ii) the rupture of the thin liquid film resulting in the formation of a three-phase contact, and (iii) the stabilisation of the particle and bubble aggregate against detachment forces.

The formation of stable particle-bubble/droplet aggregates, although not essential (e.g. Sethumadhavan et al. ${ }^{2}$ ), is regarded as having a stabilising effect on the bubbles/droplets; preventing their coalescence ${ }^{3-9}$. Obviously, the particles must have characteristics suitable for the stabilization of bubbles/droplets 
regarding their size, concentration, and hydrophobicity ${ }^{10,11}$. The coalescence of drops or bubbles is then necessarily associated with the contact of the bare interfaces, which may be due to a high capillary pressure or the presence of defects in the interfacial particle organisation. These findings generally hold for dynamic experiments ${ }^{12}$ as well as quasi-static studies ${ }^{13}{ }^{14}$. It is also noted that the tenacity of the particle-bubble/droplet aggregates is important in preventing the formation of packing defects to further delay bubble (droplet) coalescence.

Colloidal particles have been deployed in a wide range of applications to stabilise emulsions, foams, and liquid marbles $4,5,11,15-17$. One of the advantages of using colloidal particles stems from the fact that they are thermodynamically irreversibly adsorbed at the interface ${ }^{18}$. Thus, they are less susceptible to being removed from the interface compared with larger particles which are influenced to a greater extent by gravitational and inertial forces ${ }^{19}$. Although several studies have been dedicated to the effect of the size of particles on foam/emulsion stability ${ }^{15,20-22}$, more work is needed to characterise the effect of particle density.

This study reports the effect of particles of various densities on the stability of air bubbles. The particles had densities of $1.2,2.5$, and $3.6 \mathrm{~g} \cdot \mathrm{cm}^{-3}$ and were similar in size within the range $40-50 \mu \mathrm{m}$. Although smaller particles are generally preferred to stabilise interfaces, as outlined above, larger particles were used to enable their visual inspection. That is, bubbles were produced on capillary tubes, coated with particles, and their interaction recorded using a high-speed camera. Further, the technique allowed an estimation of the bubble surface oscillation dampening and the detachment of particles upon the coalescence of the bubbles. A force analysis was carried out to compare the behavior of the different particle material system.

\section{Experimental Procedure}

\subsection{Materials}

To test the relative effect of particle density, three different materials were used. Low density particles were made of a commercial cross-linked poly(methyl methacrylate), PMMA, Spheromer ${ }^{\circledR}$ CA40 (Microbeads AS, Norway). The particles were manufactured at an average diameter of $40 \mu \mathrm{m}$ and a density of $1.2 \mathrm{~g} \cdot \mathrm{cm}^{-3}$. Soda-lime glass beads were purchased from Potters Industries Pty Ltd (Melbourne, Australia). The particles had a density of $2.5 \mathrm{~g} \cdot \mathrm{cm}^{-3}$ as confirmed by pycnometry. Particles were wet sieved and the size fraction $-45+38 \mu \mathrm{m}$ was retained. The high density particles were $99.9 \%$ titanium dioxide (anatase) with an average particle size of $40 \mu \mathrm{m}$ as provided by Atlantic Equipment Engineers (Bergenfield, USA). The density of the particles was measured to be approximately $3.6 \mathrm{~g} \cdot \mathrm{cm}^{-}$

${ }^{3}$ by pycnometry, which is smaller than the value of approximately $3.9 \mathrm{~g} \cdot \mathrm{cm}^{-3}$ reported by others ${ }^{23}$. The 
water used throughout, except for the initial wet sieving of particles, was dispensed from a Milli- $Q^{\circledR}$ Pure system. The water had a resistivity of $18.2 \mathrm{~m} \Omega \cdot \mathrm{m}^{-1}$ and a surface tension of $72.8 \mathrm{mN} \cdot \mathrm{m}^{-1}$ at $20^{\circ} \mathrm{C}$.

A cleaning solution for glassware was made up of sodium hydroxide $(\mathrm{NaOH})$ (Sigma-Aldrich, $\geq 97.0$ $\%, 120 \mathrm{~g}$ ), water (120 g), and ethanol (Chem-Supply, $\geq 99.8 \%, 1 \mathrm{~L})$. Other laboratory equipment (e.g. stainless steel capillaries, stainless steel baffles, magnetic stirring bar, etc.) was sonicated first in ethanol and then in water. The cleaning of the glass particles used ammonia (Ajax Finechem, $28 \%$ ) and hydrogen peroxide (Ajax Finechem, 30\%), whereas sodium hydroxide was used to clean the anatase particles. Following the cleaning process, the hydrophobization of the glass particle surfaces was carried out using 1-octanol (Sigma-Aldrich, $\geq 99.0 \%$ ), acetone (Chem-Supply, $\geq 99.8 \%$ ), and ethanol (ChemSupply, $\geq 99.8 \%$ ). Hexane (Chem-Supply, $\geq 99 \%$ ) was used as a solvent for the measurement of contact angles. Potassium nitrate $\left(\mathrm{KNO}_{3}\right)($ Ajax Finechem, $\geq 99.0 \%)$ was used as a background electrolyte.

\subsection{Cleaning and preparation of particles}

The PMMA particles contained residual steric stabilizer. The particles were dialysed to remove this adsorbed surfactant using a cellulose dialysis membrane (molecular weight cut-off $14,000 \mathrm{~g} \cdot \mathrm{mol}^{-1}$ ). The water was exchanged twice daily for a fortnight until the surface tension of the supernatant was constant at $72.8 \mathrm{mN} \cdot \mathrm{m}^{-1}$ over the period of the measurement (i.e. $10 \mathrm{~min}$ ) as recorded on a PAT-1 tensiometer (Sinterface Technologies, Germany). The concentration of particles in the dispersion was determined by thermogravimetric analysis (TGA) (PerkinElmer Diamond TG/DTA thermal analyser). The solution was placed in aluminium pans and the analysis was performed at a temperature ranging from 20 to 560 ${ }^{\circ} \mathrm{C}$ with a ramp rate of $10{ }^{\circ} \mathrm{C} \cdot \mathrm{min}^{-1}$ and a constant flow of nitrogen gas of $30 \mathrm{~mL} \cdot \mathrm{min}^{-1}$. Polymer weight percent compositions were calculated from the mass loss up to $150{ }^{\circ} \mathrm{C}$. The concentration of the final dispersion was calculated to be $5.0 \mathrm{wt} \%$.

Glass particles were cleaned according to the methodology of Hunter ${ }^{24}$. An aqueous solution of 16.5 $\mathrm{v} / \mathrm{v} \%$ ammonia was heated to $80{ }^{\circ} \mathrm{C}$. The particles were added to the solution with $14.3 \mathrm{v} / \mathrm{v} \%$ of hydrogen peroxide and stirred for $5 \mathrm{~min}$. The cleaning solution was removed by multiple suspension/decantation stages until the $\mathrm{pH}$ returned to that of Milli- $\mathrm{Q}^{\circledR}$ water. The particles were dried in an oven at $60{ }^{\circ} \mathrm{C}$ and stored in a desiccator.

The esterification procedure consisted of refluxing $50 \mathrm{~g}$ of particles (placed in an oven at $60{ }^{\circ} \mathrm{C}$ overnight) in $130 \mathrm{~g}$ of 1-octanol in a $500 \mathrm{~mL}$ conical flask for 6 hours with a $\mathrm{CaCl}_{2}$ drying tube on the condenser to limit moisture uptake. Upon cooling, the alcohol was decanted. The particles were resuspended four times in acetone and twice in ethanol. They were then dried in a clean oven at $40{ }^{\circ} \mathrm{C}$ and stored in a desiccator. 
Rutile particles can be cleaned in a solution of sodium hydroxide within a Soxhlet apparatus ${ }^{25}$. Likewise, the anatase particles were heated in water to $85 \pm 3{ }^{\circ} \mathrm{C}$. Solid $\mathrm{NaOH}$ was added to the suspension to obtain a concentration of $10^{-2} \mathrm{M}$. Particles remained in the hot solution for $15 \mathrm{~min}$ and were then left to cool to room temperature. The solution was removed by centrifuging the suspension (Beckman Coulter, Allegra X-30 Centrifuge) in $50 \mathrm{~mL}$ test tubes at 9,000 rpm for $360 \mathrm{~min}$. The supernatant was removed and the particles were re-suspended and centrifuged a number of times until the $\mathrm{pH}$ of the water returned to that of Milli- $\mathrm{Q}^{\circledR}$ water. Fine particles were removed by decantation. The particles were dried in an oven at $40{ }^{\circ} \mathrm{C}$ and stored in a desiccator.

\subsection{Particle characterisation}

\subsubsection{Sizing}

The shape and size of the particles were characterised using scanning electron microscopy (SEM). Images of PMMA particles were obtained from a Philips XL-30 microscope whereas a Hitachi S-3400N microscope was employed for the glass and anatase particles. In each case, the samples were sputtercoated with gold.

The size distribution of dispersions of the particles was determined by laser diffraction. The size distributions of the PMMA and anatase particles were evaluated using a Mastersizer 2000 while a Mastersizer E (Malvern Instrument Ltd.) was used for the glass particles.

\subsubsection{Measurement of contact angle}

The contact angle of particles can be measured by the capillary rise of a liquid through a packed bed of particles as developed by Washburn ${ }^{26}$. The mass of liquid penetrating the packed bed of particles $(w)$ with time $(t)$ is correlated to the liquid density $(\rho)$, surface tension $(\gamma)$, viscosity $(\eta)$. It takes the form $w^{2}=\left(c t \rho^{2} \gamma \cos \theta\right) / 2 \eta$ where $c$ is a constant based on the properties of the packed bed of particles, which was determined here using hexane which has a contact angle $(\theta)$ of $0^{\circ}{ }^{27}$. The contact angle of both the anatase and the glass particles were measured using this method.

\subsection{Binary coalescence of bubbles coated with particles}

\subsubsection{Experimental setup and cleanliness}

The interaction of bubble pairs was observed by means of high-speed video using an experimental setup based on the work of Ata ${ }^{28}$. A schematic of the setup may be found in Bournival et al. ${ }^{29}$ where the sample beaker is placed in a cubic cell filled with water to avoid image distortion. All experiments were performed at $20{ }^{\circ} \mathrm{C}$ with aqueous $10^{-2} \mathrm{M} \mathrm{KNO}_{3}$ as a background electrolyte. The concentration of 
electrolyte was chosen to promote bubble coalescence ${ }^{14,30}$ as opposed to preventing (or delaying) coalescence as occurs at lower concentrations ${ }^{31,32}$.

Two independent microsyringe and pump systems were used to produce bubbles of approximately 2 $\mathrm{mm}$ in diameter at the tips of stainless steel capillaries (0.69 mm I.D. $\times 1.04 \mathrm{~mm}$ O.D.). The relative position of the capillaries may be adjusted from the translation stages on which the capillaries were mounted. The bubbles were brought together, until their surfaces are just in contact, using a programmable linear actuator (T-LA28A, Zaber Technologies Inc.). Experiments with PMMA particles were recorded with a Phantom 5 (Vision Research Inc.) while experiments conducted with glass and anatase particles were recorded with a Fastcam APX RS (Photron) high-speed camera. In all cases, a capture rate of at least 50 frames per second (fps) was used to determine the coalescence time and at least 1000 fps for the analysis of the oscillation of the bubble projected area.

The cleanliness of the setup was checked at the beginning of each experiment. Bubbles aged $120 \mathrm{~s}$ in water coalesced within $3.3 \mathrm{~ms}$ i.e. the time resolution of the experiment when recording at $300 \mathrm{fps}$. The coalescence time for the solution (including the background electrolyte) was also tested. Finally, particles were added, and bare bubbles (i.e. all particles settled) were coalesced to test for cleanliness to ensure that the continuous phase of the dispersion (PMMA) or the dry particles (glass and anatase) did not contaminate the bubble surface. Moreover, for the anatase particles for which the wettability is known to be controlled by the adsorption of atmospheric contaminants ${ }^{33}$, the coalescence time being within 1 or 2 frame intervals indicates that the surface contaminants were not solubilised in the aqueous solution. Thus, the coalescence time measurements are considered to be a more sensitive test than surface tension to detect such contamination ${ }^{30}$.

\subsubsection{Coating and coalescence of bubbles}

The PMMA particles were added as a dispersion such that the solids concentration was $0.5 \mathrm{wt} \%$. For glass and anatase particles, $1.0000 \mathrm{~g}( \pm 0.0005 \mathrm{~g})$ of particles was added to the beaker and then the coalescence of air bubbles blown at the capillary tips was examined. Bubbles covered with particles were produced by magnetically stirring the dispersion for varying times depending on the desired bubble surface coverage. The particles were then left to settle. The ageing time for all experiments was generally in the range of $1-15 \mathrm{~min}$ and is calculated from the time the bubbles (or first bubble) were produced to the time they were brought into contact.

The coverage of the bubbles by particles was calculated using a surface integral on the lower and upper halves of the bubbles ${ }^{30,34}$. ImageJ (National Institutes of Health) was used to perform the required measurements (e.g. diameter, heights, etc.). Each bubble was assessed individually, and averages were taken. The interfacial coverage of the bubbles was in most cases below the equator of the bubbles, and 
thus no particles were present in the thin aqueous film separating the interfaces upon contact. In addition, some experiments were performed with one bubble covered above its equator while the second bubble was bare, a geometry which resulted in a single layer of particles separating the contacting bubble surfaces. A schematic of the different configurations is presented in Figure 1.

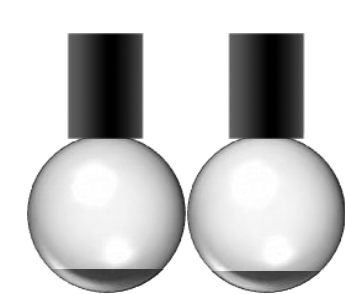

A

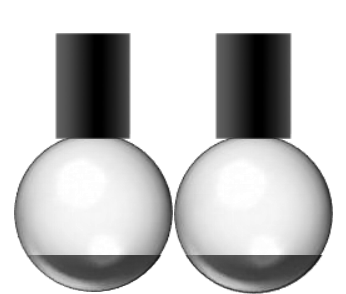

B

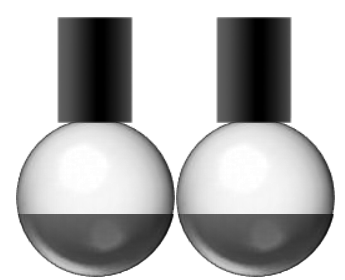

$\mathrm{C}$

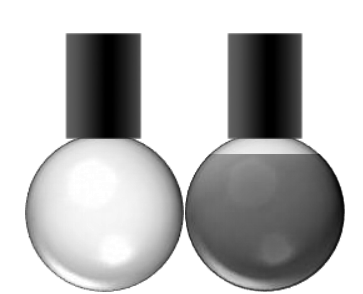

$\mathrm{D}$

Figure 1. Bubble surface coverage used in most experiments. The shaded area represents the area covered by particles. (A) Low, (B) medium, and (C) high surface coverage with respect to the contact zone, as well as (D) experiments with a single layer of particles separating the bubbles.

The analysis of coalescence dynamic and particle detachment has been described elsewhere ${ }^{30}$. A summary is presented in the Supporting Information.

\section{Particle properties}

\subsection{Scanning electron microscopy}

SEM images of the PMMA, glass, and anatase particles are presented in Error! Reference source not

found. The PMMA and glass particles were spherical in shape while the anatase particles were angular. The projected area diameter of the particles was $39 \mu \mathrm{m}$ with standard deviation $(\sigma)$ of $1 \mu \mathrm{m}$ calculated from 43 measurements $(n)$ for the PMMA; $39 \mu \mathrm{m}$ with $\sigma=4 \mu \mathrm{m}$ and $n=171$ for glass; and $51 \mu \mathrm{m}$ with $\sigma=8 \mu \mathrm{m}$ taken from 190 measurements for the anatase. The anatase particles appeared porous, which accounts for the lower than expected measured density (Section 2.1). Figure $2 \mathrm{C}$ and $\mathrm{E}$ show the broad range of particles size for glass and anatase, which was mostly likely not captured in the measurements of the projected diameter due to the small size of the samples. 


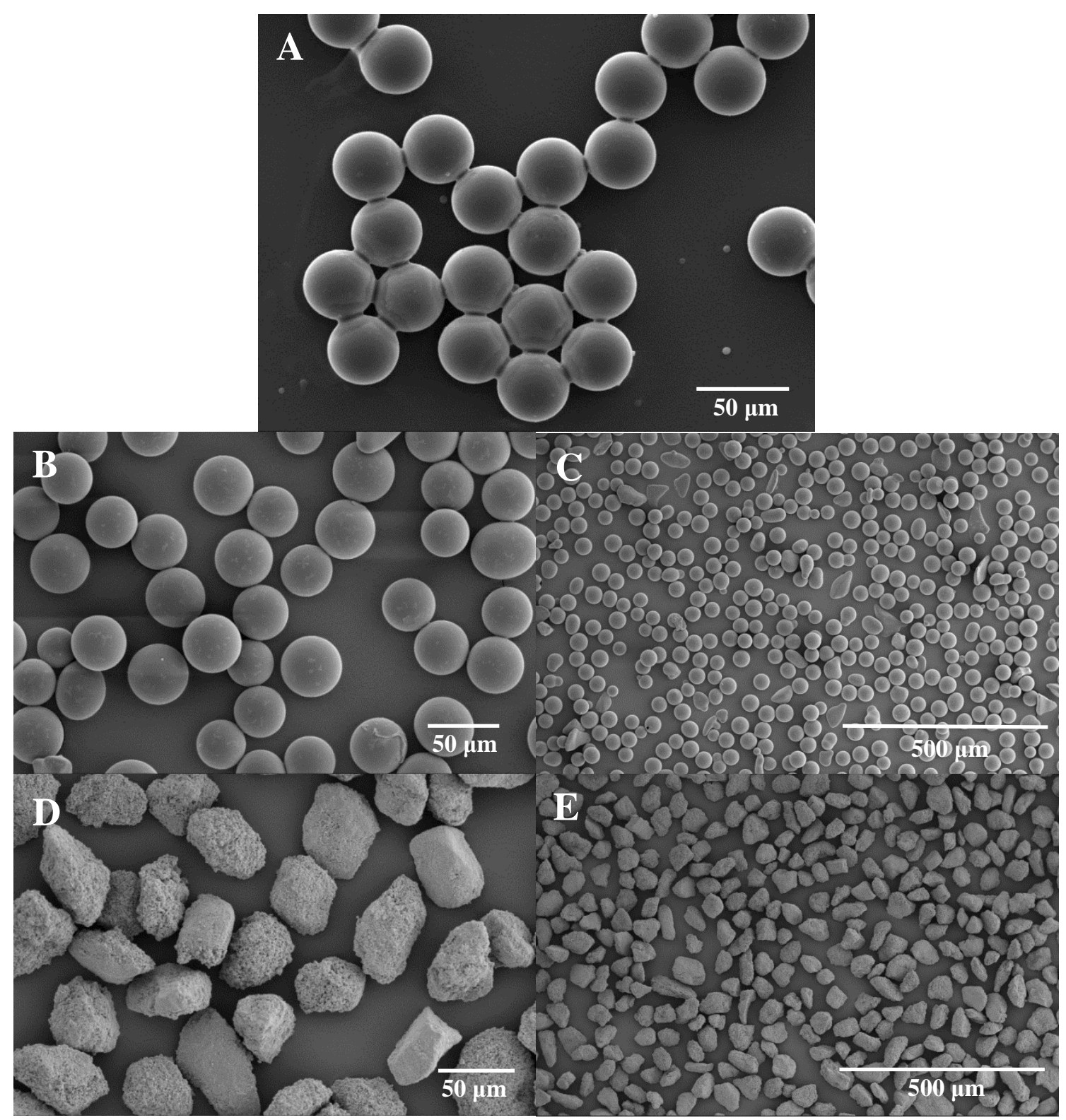

Figure 2. SEM images of (A) PMMA, (B, C) glass, and (D, E) anatase particles.

The volume frequency distributions obtained by laser diffraction are shown in Figure 3. The PMMA particles had a $50 \%$ passing size diameter $\left(\mathrm{D}_{50}\right)$ of $38.0 \mu \mathrm{m}$ and a volume-surface equivalent particle diameter $\left(\mathrm{d}_{32}\right)$ of $36.9 \mu \mathrm{m}$. The $\mathrm{D}_{50}$ and $\mathrm{d}_{32}$ were 41.2 and $39.7 \mu \mathrm{m}$ for the glass particles and 52.8 and $51.3 \mu \mathrm{m}$ for the anatase particles, respectively. All samples are relatively monodisperse. Comparing the $D_{50}$ of the samples determined on a volume basis and that of the number distribution $\left(D_{v} / D_{n}\right)$ gives a polydispersity index of 1.2 for the PMMA and anatase particles, while a polydispersity index of 1.3 was found for the glass particles. Figure 3 also include the average diameters of the particles determined from the SEM images. The projected diameters are consistent with the laser diffraction results. 


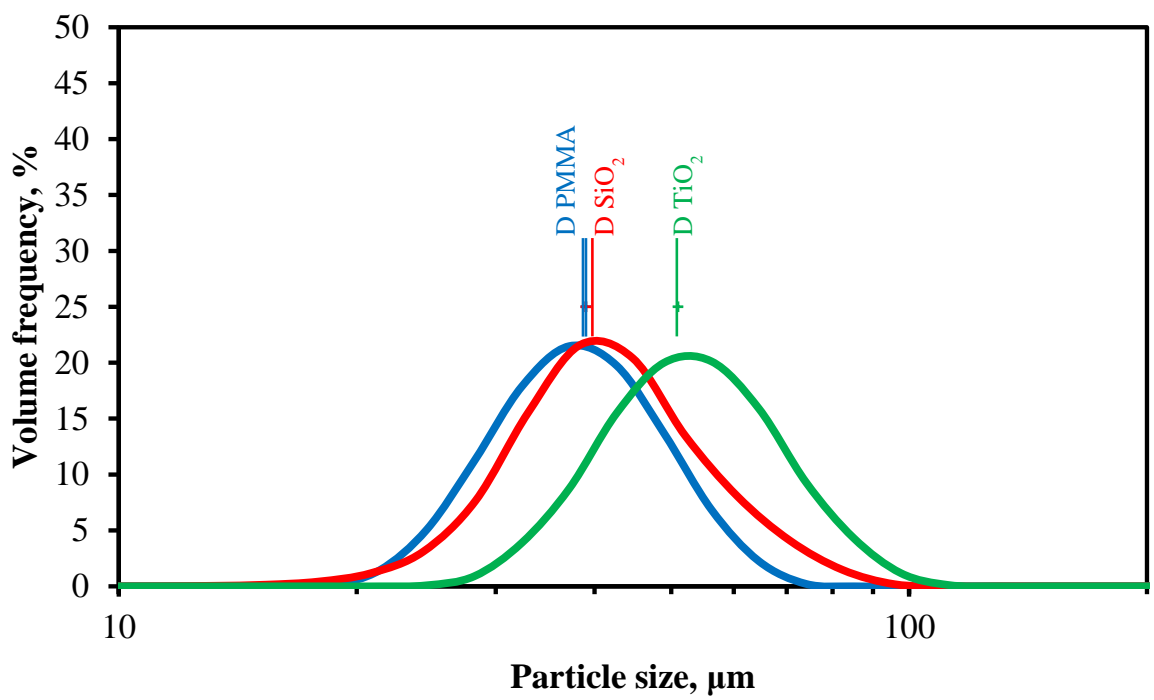

Figure 3. Volume frequency distributions of PMMA particles (blue), glass particles (red), and anatase (green). Lines mark the projected diameter derived from measurements of SEM micrographs.

\subsection{Wettability of particles}

The measurement of titania contact angle on particles is complex. For instance, Nowak and co-workers 35 used various techniques to determine the hydrophobicity of porous catalytic titania (crystal polymorph unspecified) and found the contact angle to be largely influenced by the method employed. The cleaning of rutile particles in a hot $\mathrm{NaOH}$ solution produced a contact angle of $76.6 \pm 1.4^{\circ}$ using the Washburn method with a phosphate buffered saline solution ${ }^{27}$. Due to the high surface energy of titania, the contact angle of anatase particles is also expected to be relatively high, albeit below $90^{\circ}$. The quantification of the hydrophobicity of the anatase particles was carried out through the measurement of the contact angle using the Washburn method.

The value obtained is an advancing contact angle and was found to be $64.5^{\circ}$ with a standard deviation $(\sigma)$ of $7.6^{\circ}$, which is in agreement with the control sample of MacDonald et al. ${ }^{27}$. The cleaning of the surface may lead to a contact angle of $0{ }^{\circ 33,36,37}$. Kanta et al. ${ }^{33}$ showed that following the cleaning of (amorphous) titania the contact angle starts to increase in a matter of minutes when exposed to the atmosphere due to atmospheric contaminants.

The contact angle of the glass particles was increased by covalently grafting molecules to the surface. Such an esterification procedure has been shown to produce a resistant coating of a uniform layer with a degree of hydrophobicity given by the length of the alkyl chain ${ }^{24,38-41}$. The alcohol used to esterify the glass particles was 1-octanol. The advancing and receding contact angles of a water droplet measured on an equivalent silicon wafer were found to be $73.5^{\circ}$ and $67.5^{\circ}$, respectively in the water phase ${ }^{42}$ whereas other authors ${ }^{41}$ determined an equilibrium contact angle of $75^{\circ}$ in the water phase as 
measured by the captive bubble technique. Through the Washburn method, the contact angle of the glass particles was calculated to be $79.4^{\circ}\left(\sigma=2.8^{\circ}\right)$. This value is higher than the value found by Hunter et al. ${ }^{42}$ but in relative agreement with an equilibrium contact angle of $75^{\circ}$.

The PMMA particles were observed to be relatively hydrophobic, although the contact angle was not measured here. However, Zografi and Johnson ${ }^{43}$ measured the contact angle of a smooth PMMA surface using the sessile drop technique. They found advancing and receding contact angles of $76^{\circ}$ and $52^{\circ}$, respectively, as determined in the water phase.

It should be noted that in foam stabilisation the contact angle of the particles is generally kept below 90 ${ }^{\circ}$ since particles with high contact angles act as froth breakers (i.e. anti-foaming agent). Thus, although the contact angle of the particles was found to be below $90^{\circ}$, such particles are considered 'hydrophobic', which is also the terminology adopted here. Finally, Error! Reference source not found. summarises the properties of the particles used in this study as well as particles from other studies for which results were re-analysed to complement this study.

Table 1. Summary of the particle physical characteristics and their preparation for the coalescence experiments. The table also includes particles from other studies for which results were used in the current analysis.

\begin{tabular}{|c|c|c|c|c|c|c|}
\hline Material Characteristic & $\begin{array}{c}\text { Density } \\
\mathrm{g} \mathrm{cm}^{-3}\end{array}$ & $\begin{array}{c}\text { Size } \\
\mu \mathrm{m}\end{array}$ & Shape & Cleaning & Hydrophobisation & $\begin{array}{c}\text { Contact angle in } \\
\text { the water phase } \\
(\sigma),{ }^{\circ}\end{array}$ \\
\hline PMMA & 1.2 & $\begin{array}{c}\mathrm{D}_{50}=38.0 \\
\mathrm{~d}_{32}=36.9 \\
\mathrm{D}_{\text {projected }}=39\end{array}$ & Spherical & Dialysis & None & $\begin{array}{l}\theta_{\mathrm{ad}}=76^{\mathrm{a}} \\
\theta_{\mathrm{rec}}=52^{\mathrm{a}}\end{array}$ \\
\hline Glass (finer) & 2.5 & $\begin{array}{c}\mathrm{D}_{50}=41.2 \\
\mathrm{~d}_{32}=39.7 \\
\mathrm{D}_{\text {projected }}=39\end{array}$ & Spherical & $\begin{array}{c}\text { Alkaline } \\
\text { solution } \\
\left(\mathrm{H}_{2} \mathrm{O}_{2}, \mathrm{NH}_{3}\right)\end{array}$ & $\begin{array}{c}\text { Esterification in } \\
1 \text {-octanol }\end{array}$ & $\begin{array}{c}\theta_{\mathrm{ad}}=73.5^{\mathrm{a}} \\
\theta_{\mathrm{rec}}=67.5^{\mathrm{a}} \\
\theta_{\mathrm{eq}}=75(2)^{\mathrm{b}} \\
\theta_{\mathrm{ad}}=79.4(2.8)^{\mathrm{c}}\end{array}$ \\
\hline Anatase & 3.6 & $\begin{array}{c}\mathrm{D}_{50}=52.8 \\
\mathrm{~d}_{32}=51.1 \\
\mathrm{D}_{\text {projected }}=51\end{array}$ & Angular & $\begin{array}{l}\text { Alkaline } \\
\text { solution } \\
(\mathrm{NaOH})\end{array}$ & None & $\theta_{\mathrm{ad}}=64.5(7.6)^{\mathrm{c}}$ \\
\hline Glass (coarser) ${ }^{30}$ & 2.5 & $\begin{aligned} \mathrm{D}_{90} & =92.5 \\
\mathrm{D}_{50} & =66.0 \\
\mathrm{~d}_{32} & =64.0\end{aligned}$ & Spherical & $\begin{array}{c}\text { Alkaline } \\
\text { solution } \\
\left(\mathrm{H}_{2} \mathrm{O}_{2}, \mathrm{NH}_{3}\right)\end{array}$ & $\begin{array}{c}\text { Esterification in } \\
\text { 1-butanol }\end{array}$ & $\begin{array}{c}\theta_{\mathrm{ad}}=58.0^{\mathrm{a}} \\
\theta_{\mathrm{rec}}=39.5^{\mathrm{a}} \\
\theta_{\mathrm{eq}}=43(6)^{\mathrm{b}}\end{array}$ \\
\hline Alumina ${ }^{44}$ & 3.6 & $\begin{array}{c}\mathrm{D}_{50}=24.2 \\
\text { polydisperse }\end{array}$ & Spherical & None & None & $\theta_{\mathrm{ad}}=52.4(8.8)^{\mathrm{c}}$ \\
\hline Galena ${ }^{45, \mathrm{~d}}$ & 7.6 & $\begin{aligned} \mathrm{D}_{90} & =52.0 \\
\mathrm{D}_{50} & =33.4 \\
\mathrm{~d}_{32} & =35.4\end{aligned}$ & Angular & $\begin{array}{l}\mathrm{Na}_{2} \mathrm{~B}_{4} \mathrm{O}_{7} \text { buffer } \\
\text { solution with } \\
\text { ethylenediamine } \\
\text { tetraacetic acid } \\
\text { (EDTA) }\end{array}$ & None & $\begin{array}{c}\theta_{\mathrm{eq}}=37(3)^{\mathrm{b}} \\
\text { (at pH } 8 \text { on } \\
\text { freshly cleaved } \\
\text { surface in } \\
\text { deoxygenated } \\
\text { water) }\end{array}$ \\
\hline
\end{tabular}

\footnotetext{
${ }^{a}$ Sessile drop

${ }^{\mathrm{b}}$ Captive bubble
} 
${ }^{\mathrm{c}}$ Washburn

${ }^{\mathrm{d}} 0.25$ ppm OrePrep F-549 additive

\section{Analysis}

The interpretation of the results required certain calculations to be performed. Firstly, the interaction of the particles at the interface is believed to influence the elasticity of the interface through variation in the surface pressure as demonstrated for Langmuir trough experiments ${ }^{42,46}$. The other calculations are related to the acceleration of the bubble surface, which is needed to estimate the detachment force.

\subsection{Interaction of particles at the air-water interface}

The interaction force between two partially submerged particles may be calculated following the precedent of Tarimala and Dai ${ }^{47}$ and Ata ${ }^{28}$. The calculations of the interacting force were estimated for two identical, spherical particles located at the air-water interface. The van der Waals component of the force $\left(F_{v d W}\right)$ was assumed to follow ${ }^{48}$ :

$$
F_{v d W}=-\frac{A_{e f f} r_{p}}{12 h^{2}} f(p)
$$

in which $A_{\text {eff }}$ is an effective Hamaker constant, $h$ is the separation distance, and $f(p)$ is a function, which corrects the van der Waals force for retardation effects. The effective Hamaker constant was calculated by applying a correction based on the linear fractional immersion height $(f)$ of the particles ${ }^{49}$. The effective Hamaker constant follows:

$$
A_{\text {eff }}=A_{12}+f^{2}(3-2 f)\left(A_{13}-A_{12}\right)
$$

where $A_{12}$ and $A_{13}$ are the Hamaker constants in the gas and water phase, respectively and were determined using the macroscopic theory ${ }^{50}$. The function $f(p)$ takes the form:

$$
\begin{gathered}
f(p)=(1+3.54 p) /(1+1.77 p) ; p<1 \\
f(p)=0.98 / p-0.434 / p^{2}+0.067 / p^{3} ; p>1
\end{gathered}
$$

where $p=2 \pi h / \lambda$ and $\lambda$ is the retardation length scale. The electrostatic force $\left(F_{e l}\right)$ was calculated using:

$$
F_{e l}=\frac{6 \varepsilon_{\text {air }} q_{\text {water }}^{2}}{4 \pi \varepsilon_{0} \varepsilon_{\text {water }}^{2} \kappa^{2} h^{4}}
$$

where $\varepsilon_{0}, \varepsilon_{a i r}$, and $\varepsilon_{\text {water }}$ are the permittivity of vacuum, and the relative permittivity of air and water respectively. $\kappa$ is the Debye-Hückel parameter and $q_{\text {water }}$ is the charge of the immersed section of the particle. The charge on the immersed section of the particle is: 


$$
q_{\text {water }}=2 \pi r_{p} \kappa^{-1} \sigma \tau_{\text {water }} \sin \theta
$$

in which $\sigma$ is the surface charge density, $\tau_{\text {water }}$ is the degree of dissociation of the particle surface group (e.g. silanol) in water, and $\theta$ is the contact angle. The surface charge density was approximated considering two spherical particles of low potential and follows:

$$
\sigma=\frac{\varepsilon_{0} \varepsilon_{\text {water }}\left(1+\kappa r_{p}\right) \psi_{0}}{r_{p}}
$$

where $\psi_{0}$ is the surface potential. The capillary force $\left(F_{l}\right)$ was determined using the equation of Chan et al. ${ }^{51}$, which, for the interaction of two identical particles, follows:

$$
F_{l}=-2 \pi \gamma r_{p} B^{5 / 2} S^{2} K_{1}\left(\lambda_{c} l\right)
$$

where $B$ is a Bond number $\left(B=\left(\rho_{l}-\rho_{g}\right) g a^{2} / \gamma ; g\right.$ is the gravitational acceleration, $\rho_{g}$ is the density of the gas phase), $S$ is a sphere constant $\left(S=2 D / 3-1 / 3-\cos \theta / 2+\cos ^{3} \theta / 6\right.$, where $D=$ $\left(\rho_{p}-\rho_{g}\right) /\left(\rho_{l}-\rho_{g}\right)$ is the density ratio). The expression $K_{l}$ is a modified Bessel function, which was approximated by $1 / \lambda_{c} l$, in which $\lambda_{c}=\sqrt{\left(\rho_{l}-\rho_{g}\right) g / \gamma}$ is an inverse capillary number and $l$ is the separation distance of the particles from the centres of mass (i.e. $l=2 r_{p}+h$ ).

Figure 4 shows the total interaction force between two particles of PMMA, glass, and anatase. The exact degree of dissociation of the surface groups by water was unknown but maximum values of 0.53 for glass ${ }^{52}, 0.67$ for rutile ${ }^{53}$, which was assumed as an upper limit for anatase, and 1 for PMMA were considered (dash lines). The minimum value was assumed to be 0.1 (full lines). It can be seen that the PMMA particles are expected to be attracted to one another regardless of the degree of dissociation of the surface group. On the other hand, the glass and anatase particles may be attractive or repulsive depending on the values of $\tau_{\text {water }}$. In water the degree of surface group dissociation was expected to be low in comparison to those obtained in acidic solution containing similar ions to the particles (e.g. polysilicic acid for silica). Thus all three types of particles are believed to be unstable to some extent at the air-water interface with the PMMA particles being the least stable. As such, some coagulates may be observed. 


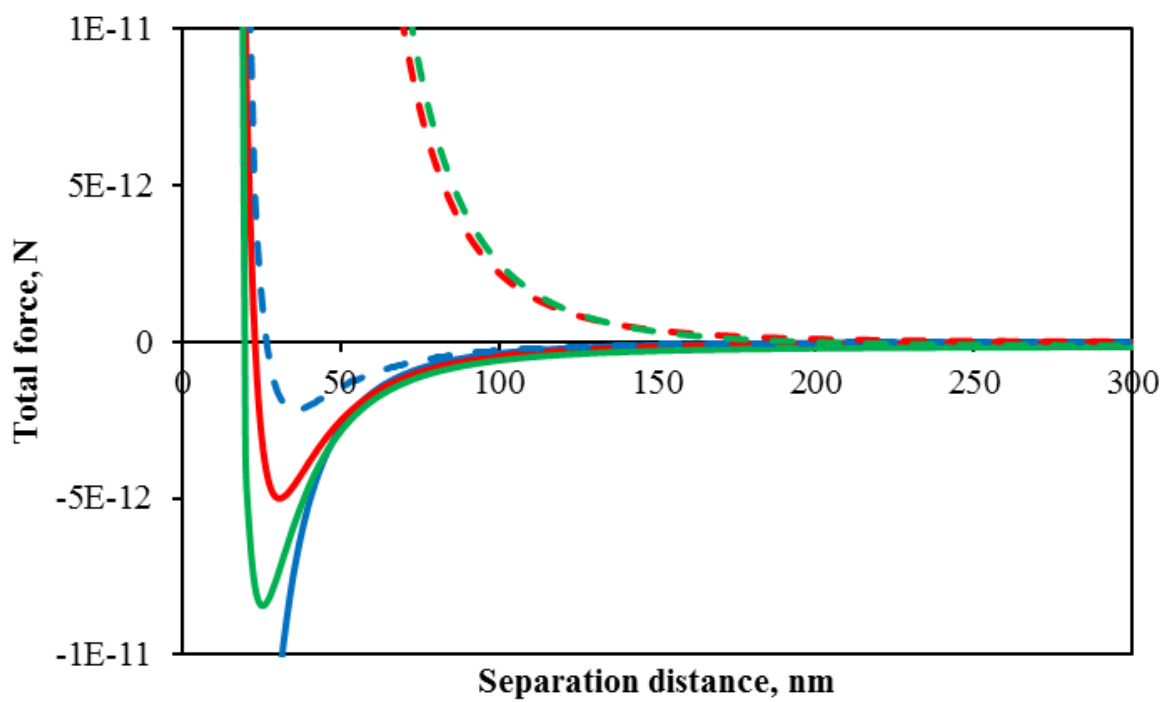

Figure 4. Interaction force between two particles of PMMA (blue), glass (red), and anatase (green). The full lines represent a degree of dissociation of 0.1 while the dashed lines used 1 for PMMA, 0.53 for glass, and 0.67 for anatase.

It is worthy to add at this stage that the calculations are for two similar particles at the air-water interface under equilibrium conditions. That is, no interpretation on the capture rate between a particle at the interface and a second particle in the liquid phase may be obtained. Although the particles may tend to agglomerate over extended time periods, there is no strong indication of multilayer formation at the bubble surface. However, as will be discussed later, some agglomeration was observed in specific cases.

\subsection{Acceleration of the bubble surface}

In the absence of surface active solutes, the dampening of the bubble oscillation is influenced by the presence of particles. However, the dampening seems not to be the driving force to expel the particles in the present system. An attempt has been made to investigate the acceleration encountered by the particles during the contraction of the interface. The horizontal distance $(d)$ between the ends of the bubble at the height of bulging was measured as a function of time. An example measurement is given in Figure 5A. The half distance $(d / 2)$ representing the displacement of one interface was plotted as a function of time (see Figure 5B - left vertical axis) and fitted with Equation 8, which is an adaptation of the equation for the coalescence dynamic (Equation S1) where $d / 2$ is the displacement of one interface and $(d / 2)_{0}$ is the initial amplitude.

$$
\frac{d}{2}=\left(\frac{d}{2}\right)_{0} \times e^{-\delta t} \times \sin \left(\omega_{0} t+\psi\right)+B
$$


In Figure 5B (right vertical axis) the acceleration of the interface was calculated from the second derivative of Equation 8 where the maximum values can be determined using the third derivative. The detachment of particles occurs upon the rapid contraction of the interface during the first cycle of the oscillation ${ }^{30}$. A maximum is found at approximately $3.7 \mathrm{~ms}$ in Figure 5B. It is hypothesised that particles which remain attached to the interface may affect the acceleration of that interface. Conversely, a high detachment of particles may not impede the motion of the interface yielding an acceleration close to that of a coalescing bare bubble. The calculated acceleration values were used in the estimation of the detachment force as presented in Section 4.3.

A

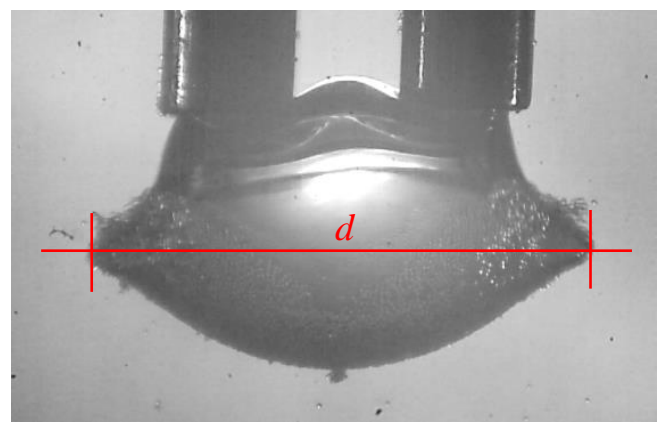

B

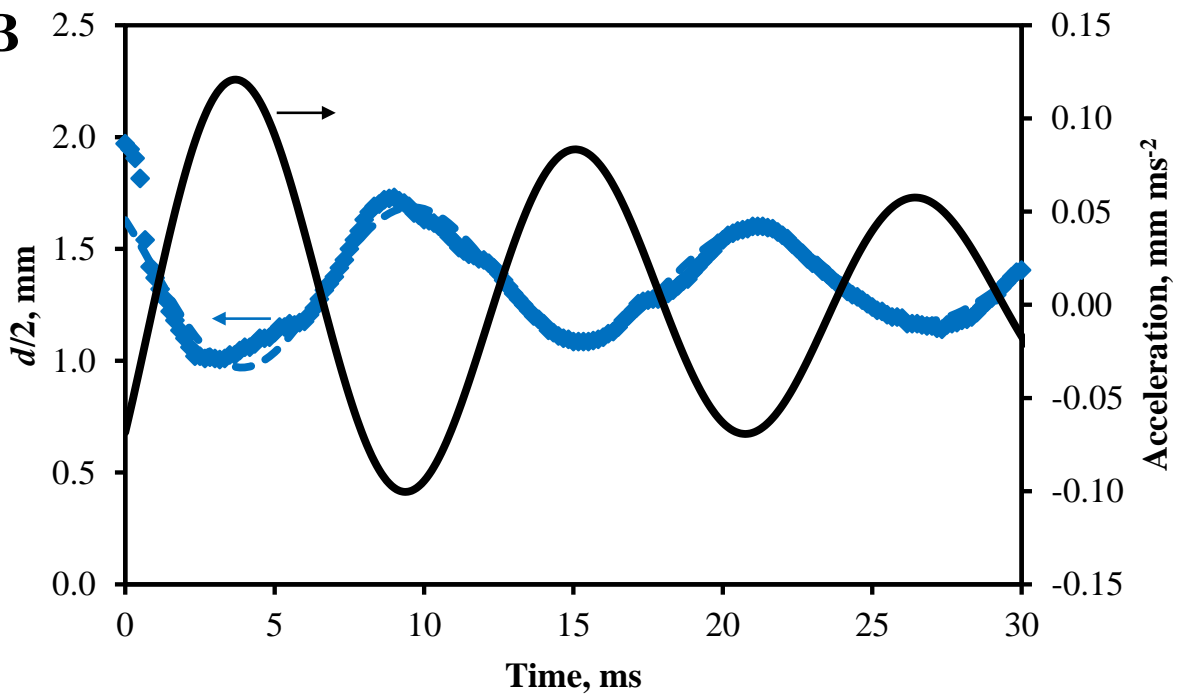

Figure 5. (A) Distance between the bubble ends at the plane of bulging during post-coalescence oscillation. (B) The displacement of one interface is plotted as a function of time ( $\diamond)$ with the fitted curve (dashed line) (left vertical axis). The full line shows the calculated acceleration based on the fitted parameters (right vertical axis).

\subsection{Comparison of forces between a particle and bubble couplet}

An attempt to quantify the tenacity of the particle-bubble aggregate, and compare them on the basis of a dimensionless number, as introduced by Schulze ${ }^{54,55}$, was made in order to get a better insight into 
the cause of the detachment of particles during the coalescence of two bubbles. The system was simplified to a single particle at a bubble surface as illustrated in Figure 6 where some key variables are also defined, which include $\alpha$ the half angle formed by the non-wetted portion of the particle and $\beta$ is the supplementary angle.

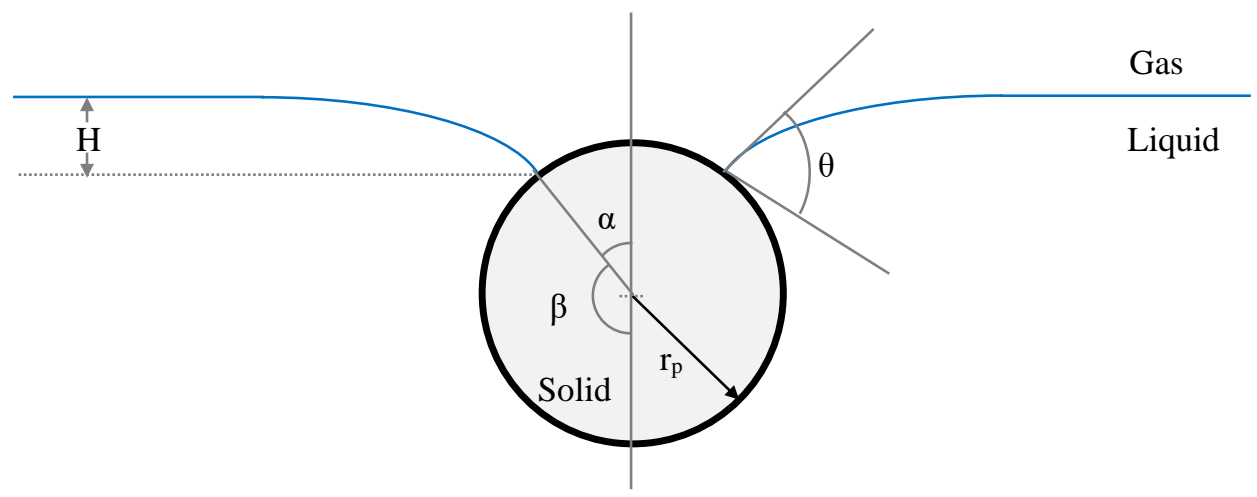

Figure 6. Schematic of a spherical particle attached to an air bubble. Drawn after ${ }^{1,56,57}$. Among the forces acting on the particles ${ }^{54-57}$ is the gravitational force $\left(F_{g}\right)$, which follows:

$$
F_{g}=\frac{4}{3} \pi r_{p}^{3} \rho_{p} g
$$

where $\rho_{p}$ is the density of the solid particle and $g$ the gravitational acceleration. The portion of the particle submersed in the liquid phase is subject to a buoyancy force $\left(F_{b}\right)$ given by:

$$
F_{b}=\frac{\pi}{3} r_{p}^{3} \rho_{l} g(1-\cos \beta)^{2}(2+\cos \beta)
$$

in which $\rho_{l}$ is the density of the liquid phase. The pressure inside the bubble is larger than in the surrounding liquid due to the curvature of the bubble as defined by the Young-Laplace equation ${ }^{58}$. This additional force, a capillary pressure force $\left(F_{p}\right)$, thus exerted on the particle may be approximated by ${ }^{56}$, 59:

$$
F_{p}=\pi r_{p}^{2}\left(\frac{2 \gamma}{r_{b}}-2 r_{b} \rho_{l} g\right) \sin ^{2} \beta
$$

where $r_{b}$ is the radius of the bubble and $\gamma$ is the surface tension. The inertial force is composed of the mass of the particle and the added mass multiplied by the acceleration of the particle $\left(b_{m}\right)^{57}$. In this particular context, the acceleration was taken as the maximum acceleration as found by the horizontal displacement of the interface as shown in Figure 11. The inertial force $\left(F_{i}\right)$ takes the form:

$$
F_{i}=\frac{\pi r_{p}^{3}}{6} b_{m}\left(8 \rho_{p}+\rho_{l}\left(2+3 \cos \alpha-\cos ^{3} \alpha\right)\right)
$$


Finally, unlike the other forces the capillary force $\left(F_{c}\right)$, which arises from the tension of the interface on the three-phase line of contact, keeps the particle at the interface.

$$
F_{c}=2 \pi \gamma r_{p} \sin (\alpha) \sin (\theta-\alpha)
$$

The formation of a meniscus due to the deformation of the bubble surface causes the three-phase line of contact to lie below the plane formed by the undeformed interface. This phenomenon gives rise to a hydrostatic force $\left(F_{h}\right)$, which is dependent upon the depth of the meniscus depression $(H)$.

$$
F_{h}=\pi r_{p}^{2} \rho_{l} g L H \sin \alpha
$$

where $L$ is the capillary length and is defined as:

$$
L=\sqrt{\frac{\gamma}{\rho_{l} g}}
$$

The depression of the meniscus may be approximated from the relationship proposed by Nguyen and Schulze ${ }^{58}$ :

$$
H=2 L \sin \left(\frac{\theta-\alpha}{2}\right)\left(1+\frac{L}{r_{p} \sin \alpha}\right)^{-1 / 2}
$$

The modified Bond number $\left(B o_{m}\right)$, which is an expansion of the Bond (or Eötvös) number, is simply defined as the ratio of the detachment forces over the attachment forces, or mathematically,

$$
B o_{m}=\frac{\left(F_{g}-F_{b}\right)+F_{p}+F_{i}}{F_{c}+F_{h}}
$$

Theoretically, a $B o_{m}$ of 1 indicates that the attachment and detachment forces are equivalent. A $B o_{m}<$ 1 means that the attachment forces dominate the force balance while a $B o_{m}>1$ signifies that the detachment of particles is expected.

\section{Results and Discussion}

\subsection{Stability of bubbles covered by particles}

The stability of bubbles covered with different particulate materials was assessed by measuring the coalescence time. Results, which are reported in Table 2, were divided into two groups composed of measurements where particles are not in the contact zone (configurations A, B, and C in Figure 1) and with one layer of particles separating the bubbles (configuration D in Figure 1). In the absence of particles in the contact zone coalescence always occurred. These coalescence times are relatively short compared to those obtained when one layer of particles was present. For the latter configuration, the coalescence times increased with decreases in the density of the particulate materials. As coalescence 
time is a measure of bubble stability, it is not surprising that the probability of the coalescence event followed a similar trend.

Table 2. The range of coalescence times and coalescence probability (brackets) of bubble pairs covered with particles.

\begin{tabular}{|c|c|c|}
\hline Material & No particles in the contact zone & $\begin{array}{c}\text { One layer of particles in the contact } \\
\text { zone }\end{array}$ \\
\hline PMMA & $0.1-0.6 \mathrm{~s}(100 \%)$ & $0.7-336 \mathrm{~s}(78 \%)$ \\
\hline Glass & $0-0.018 \mathrm{~s}(100 \%)$ & $0.5-90 \mathrm{~s}(83 \%)$ \\
\hline Anatase & $0-0.02 \mathrm{~s}(100 \%)$ & $0.02-2 \mathrm{~s}(100 \%)$ \\
\hline
\end{tabular}

Studies ${ }^{6,10,12}$ have shown that for bubbles and emulsions, the formation of defects such as the lowering of the particle packing density (i.e. expansion of the interfacial area) or the displacement of particles is the most likely explanation of the instability of the bubble pairs separated with a particle layer. The capillary pressure needed to contact the bubble surfaces is not expected to be overcome when the particle layer is intact under the current experimental conditions. The displacement of particles may lead to a decrease in the packing density of the particles, which may cause the coalescence of the bubbles through: (i) the exposure of areas of bare bubble surfaces, or (ii) the exceeding of the maximum capillary pressure. The latter is reduced due to the increase in the spacing between particles ${ }^{60,61}$.

The PMMA particles appeared to form a more resistant coating which prevented coalescence more often, and offered a prolonged stability against coalescence. On the other hand, the anatase particles were a poor bubble stabilizer. The effect of the density of the particles may not be obvious, but other results (e.g. Figure 9) showed that the denser particles were more easily removed from the interface. This observation was also confirmed through the comparison of the forces acting on the particles, which indicates that anatase particles are more prone to detachment (as discussed later - see Figure 12). Thus, it can be inferred that packing defects may be more readily created with anatase (and glass) particles than with PMMA particles. The presence of packing defects was deduced through visual inspection as depicted in Figure 7. Figure 7B also illustrates how a lateral force induced by the second bubble may push particles off the interface, which will then lower the packing density around the contact point. In the case of the glass particles, the force between the particles in the water phase was such that they remained agglomerated. This removal of particles creates the packing defect required to promote bubble coalescence as depicted in Figure 7. 

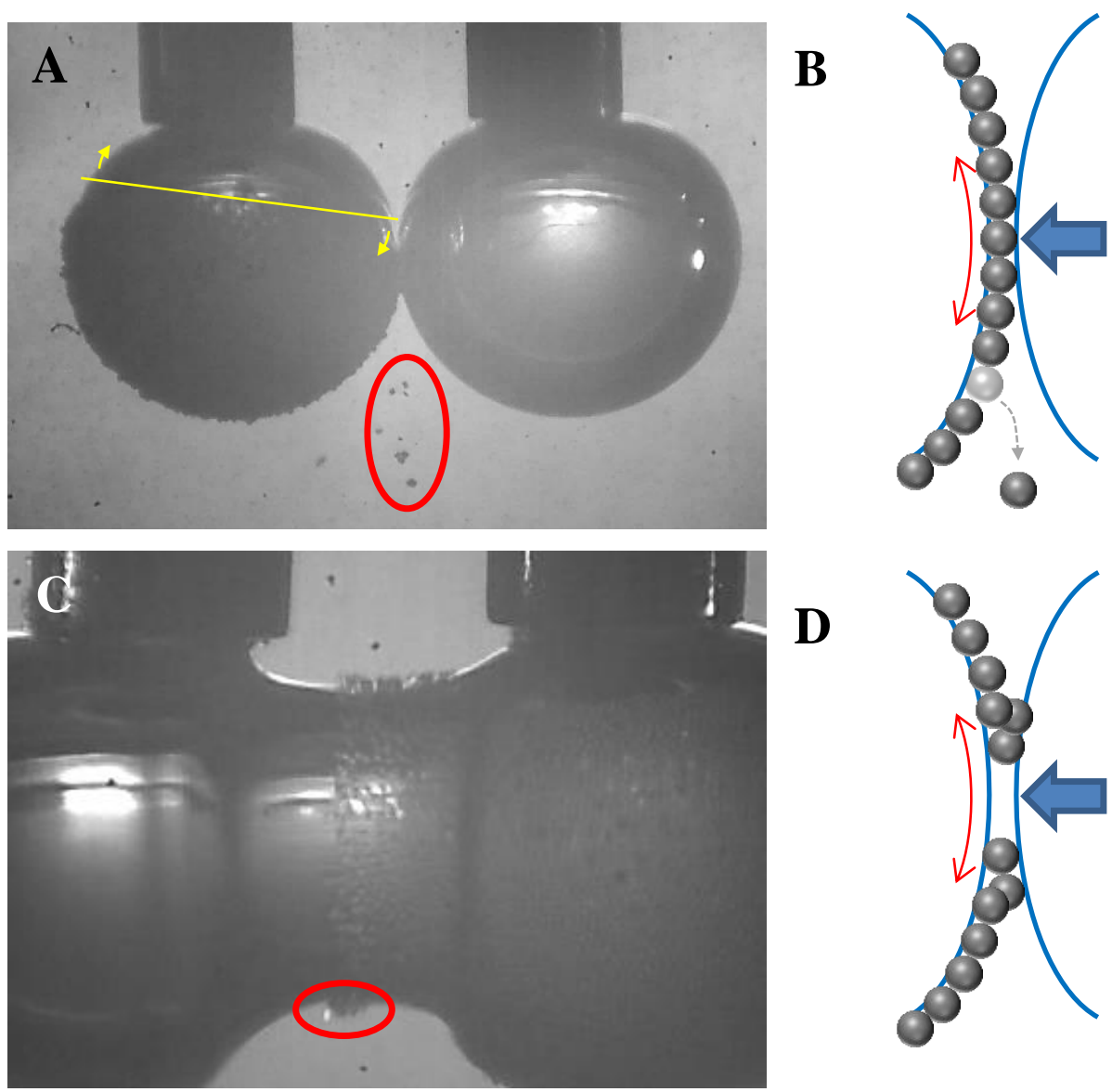

Figure 7. (A) Visual evidence of the anatase particle coating movement (yellow line) and the detachment of particles during the contact and the re-organisation of the particles (circled in red). The outer diameter of the capillary tube is $1.04 \mathrm{~mm}$ and is used as an effective scale bar. (B) Schematic of the lateral force caused by the contact of the second bubble. The lateral force may cause particles to detach. In $(C)$ there is evidence of particles agglomeration upon the rupture of the thin liquid film. The lateral force exerted by the second bubble causes the removal of particles from the interface, which agglomerates to the surrounding particles as shown in (D).

The formation of a defect sufficient for the destabilisation of the contacting bubbles may take some time, as shown by Bournival et al. ${ }^{10}$, and explains the large coalescence time reported in Table 2 when a layer of particles is present between the two bubble surfaces. In terms of mechanisms which may generate packing defects, the lateral displacement of the particles combined with the removal of particles from the interface is a plausible hypothesis as evidenced in Figure 7. This lateral displacement of the whole coating may be accompanied by a localised displacement of particles near the contact point (see Supporting Video - displacement of glass [finer glass, $\theta_{\mathrm{ad}}=79.4^{\circ}$ ]). The force analysis presented in Section 4.1 may partly explain the greater stability of the bubbles covered with PMMA particles as a larger force is required to move particles away from each other and create packing defects. PMMA particles are believed to be difficult to displace from the interface (see Figure 12). In addition, Figure 4 revealed that the particles are strongly attracted to each other at the interface, which may lead to the formation of a coherent network of particles resistant to the formation and propagation of defects. That is to say, the phenomena observed for glass and anatase (illustrated in Figure 7) were not observed for 
the experimental system containing PMMA particles. It should also be noted that due to the configuration of the experimental systems, gravitational forces and a radial force ${ }^{62}$, which arises from the packing of particles, may still overcome the total (repulsive) force shown in Figure 4. It may be speculated that the displacement/sliding of particles is easier for glass and anatase, e.g. Supporting Video. In addition, the fact that glass and anatase are denser may lead to the increased likelihood of particles detaching from the bubble surface, e.g. Figure 7.

\subsection{Effect of bubble loading on the bubble surface properties and particle detachment}

The analysis of the interfacial properties of the bubble during coalescence may be performed by modelling the projected area as a function of time ${ }^{10,29,30}$. Figure 8 compares the dampening of the bubbles during coalescence as a function of the coverage of the bubble by particles. The increased loading of the bubble caused an increase in the dampening of the bubble oscillation in the case of PMMA and, less significantly, for the glass particle system. It has been noted that geometrically, the particle coverage of the bubbles need to be relatively high in order to possibly affect the bulging of the bubble periphery, which occurs approximately at the same height as the point of rupture of the thin liquid film ${ }^{10}$. Thus, the dampening of the oscillation may be a function of the initial coverage of the bubbles ${ }^{14}$, especially when the particles remain on the surface. The damping coefficient appears to remain relatively constant for the anatase system regardless of the initial fractional coverage of the bubble by particles, which may be caused by the high degree of detachment of particles.

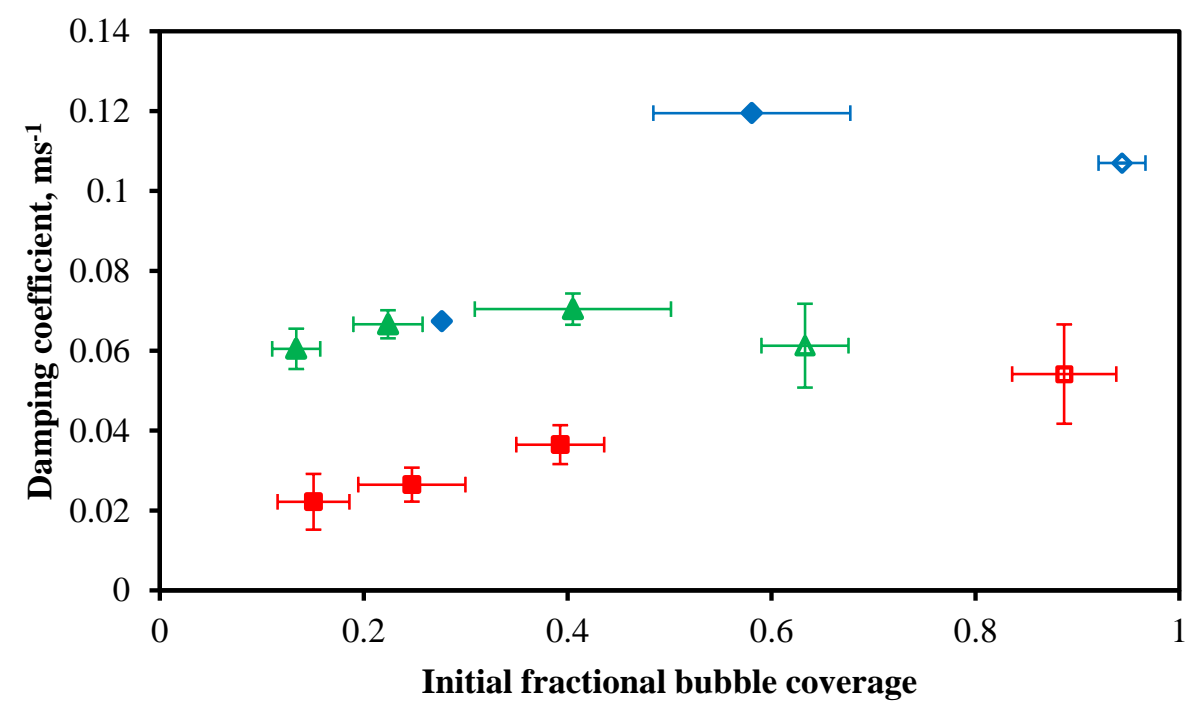

Figure 8. Effect of the initial fractional coverage of the bubbles by particles on the dampening of the coalescence oscillation. The closed markers represent the coverage of two bubbles (e.g. configurations $\mathrm{A}-\mathrm{C}$ in Figure 1) whereas the open markers show the coverage of one bubble (configuration D in Figure 1). Results are presented for $(\diamond)$ PMMA, ( $\bullet$ ) glass, and $(\Delta)$ anatase particles. 
The effect of the initial coverage of the bubbles on the detachment of particles is shown in Figure 9. Except in the case of a single bubble covered above its equator by glass particles (configuration D in Figure 1), the detachment of particles appeared to be unaffected by the initial bubble loading.

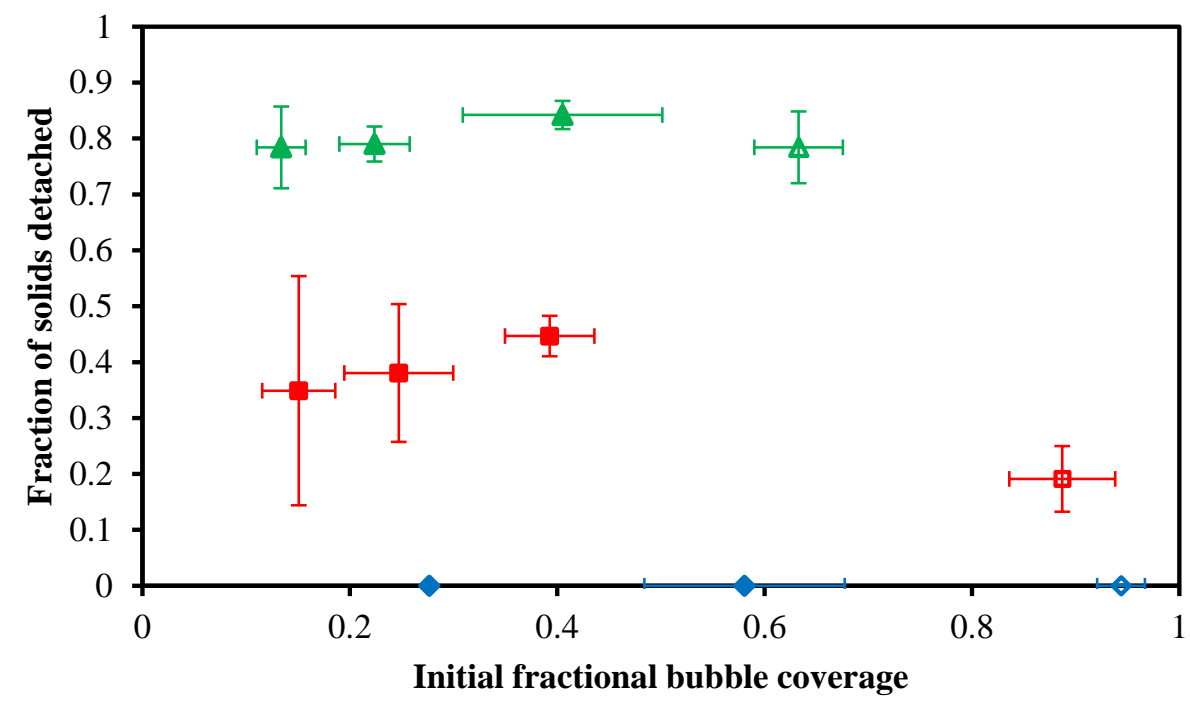

Figure 9. Effect of the initial fractional coverage of the bubbles by particles on the fractional detachment of particles upon bubble coalescence. The closed markers represent the coverage of two bubbles (e.g. configurations A - C in Figure 1) whereas the open markers show the coverage of one bubble (configuration D in Figure 1). Results are presented for ( $\diamond$ PMMA $\left(\rho_{p}=1.2 \mathrm{~g} \mathrm{~cm}^{-3}\right)$, (घ) glass $\left(\rho_{p}=2.5 \mathrm{~g} \mathrm{~cm}^{-3}\right)$, and $(\Delta)$ anatase $\left(\rho_{p}=3.6 \mathrm{~g} \mathrm{~cm}^{-3}\right)$ particles.

The damping coefficient may be used to predict the ability of the bubble surface to retain particles as previously reported for the detachment of glass particles in cetyltrimethylammonium bromide (CTAB), ${ }^{13}$ or frother solutions ${ }^{30}$, and for galena particles in frother solutions ${ }^{45}$. In these studies the effect of the dampening of the oscillation related to the detachment of particles (i.e. comparing the same experimental system). However, the establishment of a relationship between the damping coefficient and the degree of particle detachment has not previously been attempted for different particulate systems. For the sake of generality, data from previous studies has been added to those measured in the current study. The characteristics of these additional particles are reported in Error! Reference source not found., which comprise alumina, galena, and glass particles. The glass particles were coarser and less hydrophobic than those used in this study while of similar density. Figure 10 shows that the lower damping coefficients are generally associated with a higher fractional detachment of particles with the exception of the finer $(39.7 \mu \mathrm{m})$ glass particles. The transition, in Figure 10, may occur at a damping coefficient of approximately $0.06-0.08 \mathrm{~ms}^{-1}$, which is interestingly close to the value found for the reduction in detachment of glass particle by adsorbed frothing agents ${ }^{30}$. Thus, there may be an oscillation dampening, characteristic to two coalescing bubbles, in which particles are more likely to detach regardless of their properties. However, the relationship between particle detachment and 
oscillation dampening is not straightforward when considering the effect of the different particle materials on the latter which is distinct for the different particles (Figure 8).

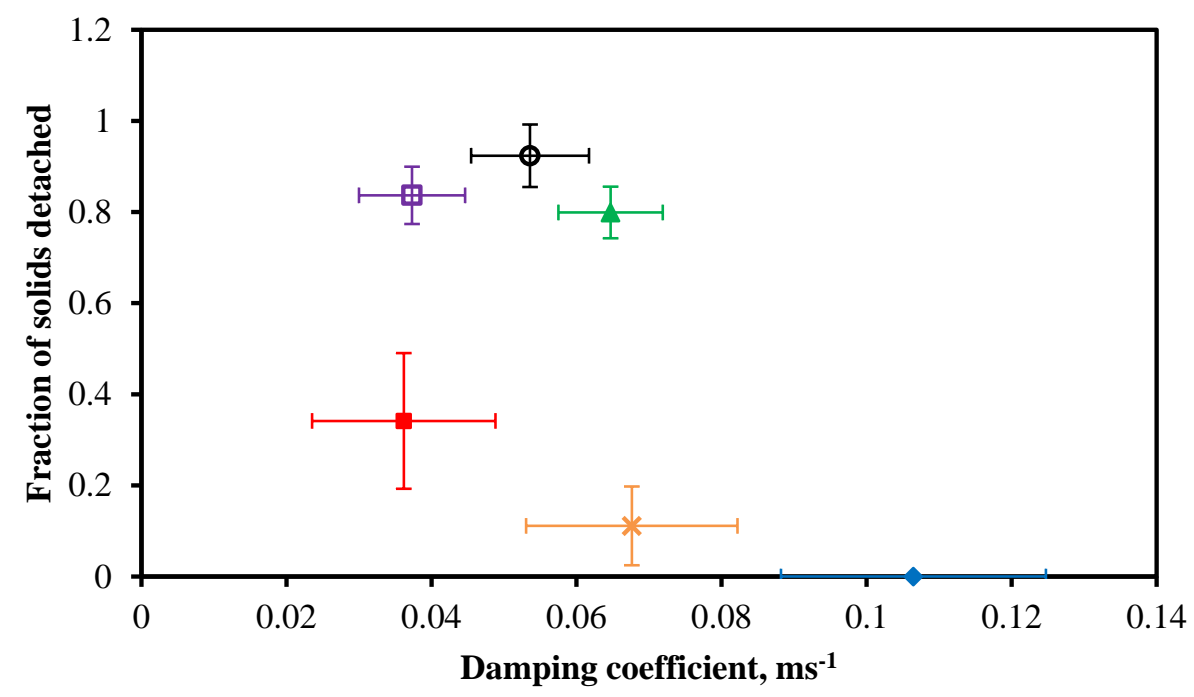

Figure 10. Fractional detachment of particles as a function of the damping coefficient of the oscillation of the projected area of the coalescing bubble. Experiments were performed with $(\diamond)$ PMMA, ( $\square$ ) glass (finer, $\theta_{\mathrm{ad}}=73.5^{\circ}$ ), and $(\Delta)$ anatase particles. Also included are results from other studies using $(\square)$ glass (coarser, $\left.\theta_{\mathrm{ad}}=58.0^{\circ}\right),(\times)$ alumina, and $(\circ)$ galena.

The comparison of the two experiments with glass particles in Figure 10 may shed light on the many factors influencing the damping coefficient. Between the two sets, the hydrophobicity and the size of the particles were different, while the density remained constant. The results show that the damping coefficients are nearly identical for the two glass samples, which however yielded convincingly different detachment values. The larger and less hydrophobic particles detached in greater numbers than the smaller more hydrophobic particles as expected but the detachment cannot here be attributed to the mass of particles remaining on the surface. Smaller particles are known to produce a larger damping coefficient of the bubble oscillation ${ }^{10,13}$ most probably through increases in the elasticity of the surface ${ }^{63-65}$. In addition, the dampening of the oscillation of the bubble may be found to increase with increases in the degree of hydrophobicity of the particles ${ }^{10}$. Thus, the damping coefficient found for the smaller $(39.7 \mu \mathrm{m})$ and more hydrophobic $\left(73.5^{\circ}\right)$ particles was found to be unexpectedly low.

During the oscillation of the bubble surface, the particles on the surface may affect the oscillation through the formation of a connected particle network ${ }^{66}$, viscous energy dissipation (i.e. drag) ${ }^{28}$, and a change in the properties of the composite surface made up of particle and bubble surfaces ${ }^{67}$. It is difficult to measure these properties when the mass of particles is changed during the dynamic oscillation. However, it has been noted that most of the particles detach during the first contraction of the bubble. Thus was hypothesized that a relationship may exist between the detachment of particles and the maximum acceleration, which, for the bubble interface, occurs in the first cycle when the bubble 
is fully contracted (Figure 5B). Figure 11 shows the maximum acceleration of the interface as a function of the detachment of particles, as outlined in Section 4.2. The figure compares the average maximum acceleration of the bubble interface from the different systems with a system without particles (dash line).

The fact that the particle-bubble surface composite changed due to the detachment of particles does not appear to systematically influence the acceleration of the bubble surface. Such information may be too sensitive to be detectable through a change in the displacement of the interface as depicted in Figure 5A. It should also be noted that the detachment of particles itself may affect the total energy of the system knowing that thermodynamically an energy decrease is calculated for the adsorption of particles at an air-water interface ${ }^{68,69}$. Conversely, the surface properties of the bubble surface may be explained by the detachment of particles. It has been shown that the dampening of the bubble surface oscillation is influenced by the loading of particles ${ }^{14}$. It was speculated that the high detachment of anatase particles observed here may limit the dissipation of energy through viscous resistance. On the other hand, the PMMA particles, which tended to remain at the bubble surface, were subject to the smallest acceleration force. However, no clear correlation emerged between the fractional detachment of the particles and the maximum acceleration of the bubble surface. Nevertheless, the average acceleration found for the different systems was used in the detachment analysis outlined in Section 4.3 and presented in Section 5.3.

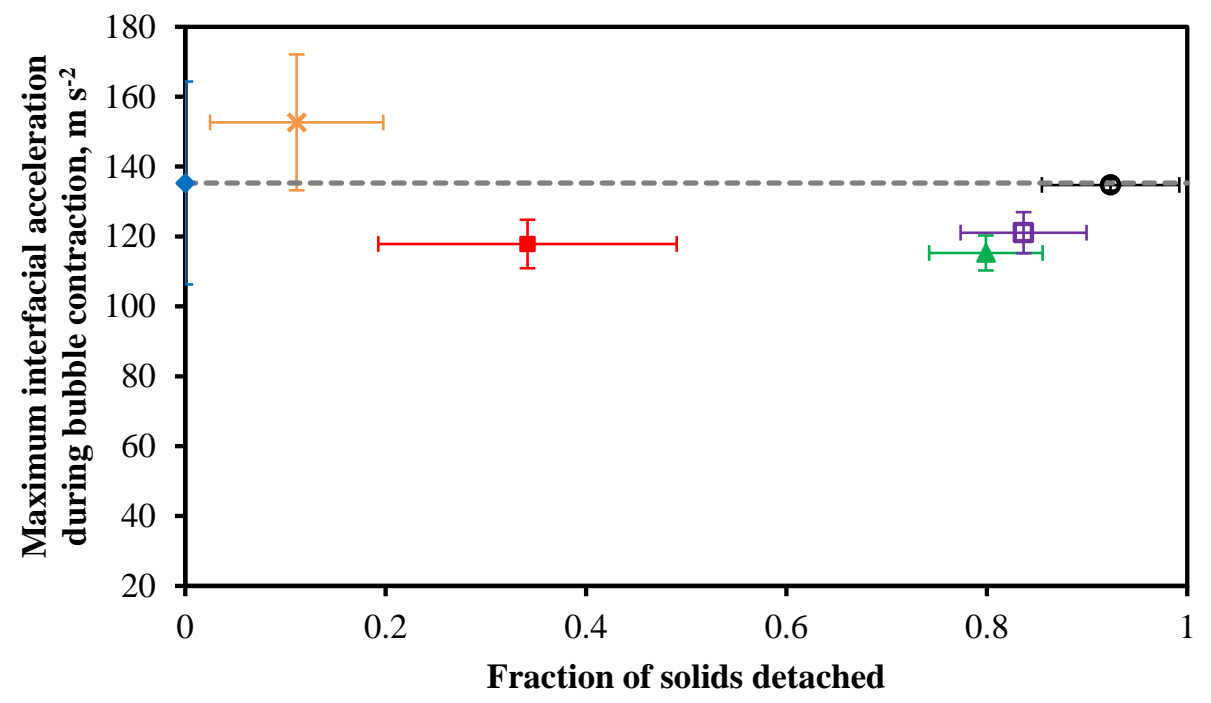

Figure 11. Effect of fractional particle detachment on the maximum acceleration of the projected area during the first contraction of the interface upon the coalescing of bubble pairs. Experiments were performed with ( $\diamond$ PMMA, $(\bullet)$ glass (finer, $\theta_{\mathrm{ad}}=73.5^{\circ}$ ), and $(\Delta)$ anatase particles. Also included are results from previous studies using $(\square)$ glass (coarser, $\theta_{\mathrm{ad}}=58.0^{\circ}$ ), $(\times)$ alumina, and $(\circ)$ galena and are all compared to the average for bare bubbles coalescing in water (dashed line). 


\subsection{Detachment analysis in terms of bubble surface properties}

Figure 12 shows the effect of the force balance, that is the modified Bond number, on the fractional detachment of particles from the bubble. It can be observed in Table 1 that the value of the contact angle of the particles may be affected by the method employed. The horizontal error bars in Figure 12 show the variation in the modified Bond number for a change of $\pm 5^{\circ}$ in the advancing contact angle. For the different materials, a transition can be observed with low detachment for, generally speaking, smaller, low density particles and higher detachment for larger, higher density particles. As such, the results are following the expected trend except for the fact that the transition was observed to be two orders of magnitude lower than anticipated and seemed to be unaffected by slight variations in the contact angles of individual particles (i.e. calculations not affected by the contact angles measuring technique). It should be emphasized that the force balance ${ }^{1,55}$ which lead to the development of the modified Bond number was based on a single spherical particle. The calculated capillary force is approximately two orders of magnitude larger than the other detaching forces, which explain the low values of the modified Bond number. It is believed that the attachment force estimated using Equation 13 were an overestimation from the fact that there is more than one particle, and the particle surface is not homogeneous. Other factors may have influence the magnitude of the modified Bond number as follows.

Particles which are positioned higher on the bubble may exert a radial force on the lower particles ${ }^{62,70}$, which may act as an effective detachment force. Moreover, it was found that angular particles had a broader detachment probability distribution than spherical particles. These results were corroborated by Goel and Jameson ${ }^{56}$ who observed a 10 to $15 \%$ increase in the detachment of angular particles compared to nearly spherical particles. However, when comparing similar wetted perimeters, sharp edges may accentuate the capillary force ${ }^{71}$. The pinning of the interface onto the particle due to surface roughness (or even superficial pores) may give rise to a larger attractive force. Thus, particles like the anatase (see Error! Reference source not found.) may appear more hydrophobic. It should be noted that such pinning may be followed by a slip of the three-phase line of contact and destabilisation of the particle-bubble aggregate ${ }^{72-74}$. In addition, the orientation of the particles, if irregular, may determine whether the pinning promotes particle detachment. Thus, the low transitional value noted in Figure 12 may be partly imputed to superficial defects. However, in the specific case of the anatase and galena particles, it appears that the trend corresponds to that observed with the spherical glass particles. Thus, any pinning is considered to be negligible compared to other forces. 


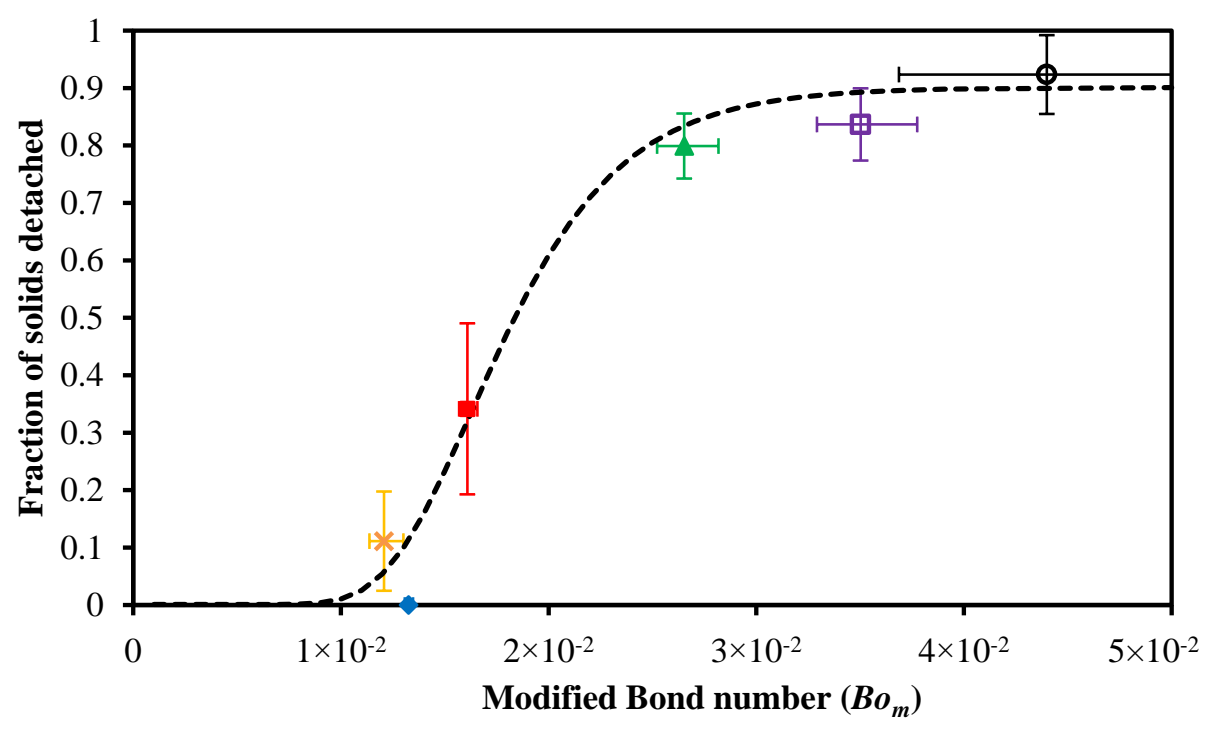

Figure 12. Fractional detachment of particles expressed as a function of the modified Bond number. Experiments were performed with ( $\diamond$ ) PMMA, ( () glass (finer, $\theta_{\mathrm{ad}}=73.5^{\circ}$ ), and $(\Delta)$ anatase particles. Also included are results from other studies using $(\square)$ glass (coarser, $\left.\theta_{\mathrm{ad}}=58.0^{\circ}\right),(\times)$ alumina, and (०) galena. The dashed line shows the general trend. The horizontal error bars represent the effect of a change of $\pm 5^{\circ}$ in three phase contact angle on the modified Bond number.

More significant is the finding by Nutt ${ }^{75}$ that the centrifugal forces needed to detach a raft of particles was considerably less than the force required to remove a single particle. A single particle, as calculated in section 4.3, is subject to an absolute inertial force ${ }^{57}$. It is hypothesised that the formation of rafts due to attractive inter-particle forces ${ }^{76}$ underestimated the actual mass of the particle while considering the raft as a single entity. Stevenson et al. ${ }^{57}$ also noted the finite response of the three-phase contact line (formed by $\alpha$ in Figure 6). The three-phase contact line is necessary to establish an equilibrium position and retain the particles at the interface ${ }^{77}$. The time lag of the three-phase contact line due to the high frequency oscillation may lead to the premature detachment of the particles since the three-phase contact line is not in an equilibrium position. On a similar note, the contact angle hysteresis has been shown to influence the detachment of a particle ${ }^{78}$ and is not accounted for in force balances e.g. ${ }^{55,56 \text {, }}$ ${ }^{58,79}$. Finally, a knowledge of the local forces is required to estimate properly the detachment force ${ }^{10}$. In other words, phenomena such as particle motion on the surface of the bubble or the presence of surface waves ${ }^{80,81}$ are not incorporated in the estimated acceleration of the particles, which was taken as the acceleration of the interface (see Figure 5A). It is speculated that the method presented in section 4.2 may be an underestimation of the actual acceleration acting on the particles.

The trend shown in Figure 12 facilitates comment on the relative magnitude of the forces involved. Among those considered, the hydrostatic force may rightly be ignored for the current particulate systems ${ }^{54,56,57}$. The major detachment force arose from the pressure inside the bubble exerting a force 
on the particle $\left(F_{p}\right)$. While investigating different particulate systems, the main parameters affecting this force are the size and the hydrophobicity of the particles. Similarly to the inertial force, the capillary pressure force may be underestimated. The local pressure inside the bubble is unknown during the dynamic oscillation process. For instance, the radius of curvature is changing with time and in the different location on the surface of the bubble (see Figure 5A bubble contour). Thus, the force exerted by the capillary pressure on the particle may also be more elevated.

The other major force contributing to the detachment of the particles was related to the density (and size) of the particles. Whereas the weight of the particles themselves $\left(F_{g}\right)$ was not especially significant, the added mass due to the inertial forces, which like the gravitational force combines the density and size of the particles, was one order of magnitude higher than the latter and comparable to $F_{p}$ in terms of magnitude (except for the PMMA where $F_{a}$ was an order of magnitude lower). While other investigations $56,59,75,82$ indirectly studied the effect of the gravitational and inertial forces through a change in the size of the particles, the role of density may here be discussed in terms of the PMMA. It is, in fact, interesting to note that although the modified Bond number of the PMMA rests between that of the alumina and the $39.7 \mu \mathrm{m}$ glass particles the fractional detachment remained negligible. It is theorized that the inertial force may contribute to the motion of the three-phase contact line, which subsequently caused the destabilisation of the bubble-particle aggregate.

The potential importance of the density of the particles thus highlighted may be important in the selection of materials to stabilise foams, emulsions, and liquid marbles. A considerable number of studies has shown that the stability of such dispersions is affected by the concentration of particles ${ }^{7,42}$, 60,83. Assuming that the attachment/adsorption of particles is independent of the density of the particles, the lower density particles were shown to be more effective at preventing bubble coalescence (Section 5.1). In addition, due to the lower detachment of particles, the coverage of the coalesced bubble is higher in the case of PMMA. As such, it is suggested that the stability of the coalesced bubble may be increased compared to those of other particulate systems. Thus, the selection of relatively low density particles, relative to the continuous phase, may be a key factor in the handling of dispersions requiring high stability against coalescence.

The finding is also significant for industrial processes such as froth flotation. In froth flotation, valuable particles are promoted to attach on air bubbles, which rise to the surface to form a froth. The froth is collected to recover the valuable materials. The bubbles in such a system are transiently stable, and bubble coalescence regularly takes place. To optimise the recovery of particles, the probability of a particle to detach should be low ${ }^{84}$. A few strategies may be put in place to reduce this probability. The attachment force may be increased by increasing the hydrophobicity of the particles, which should be kept below $90^{\circ}$. Alternatively, the detachment force may be decreased for dense particles through size reduction to reduce the inertial force experienced by the particles, although this would be a less 
preferable approach due to the high cost associated with grinding. Due to the added cost of grinding, another option is to reduce the detachment force by designing flotation devices, which are more quiescent ${ }^{85}$, or modify the bubble interfacial properties through the addition of surface active reagents 30.

\section{Conclusions}

The stability of bubbles loaded with particles of different densities was tested through the pairwise interaction of bubbles. Coated bubbles were brought into contact, and the stability of the bubbles was evaluated by their coalescence probability and the time needed for coalescence to occur. The surface properties of the bubbles as well as the stability of the bubble-particle aggregate were estimated during the coalescence of the bubble pairs.

The less dense particles tended to form a more resistant coating against coalescence. In fact, denser particles were seen to be dislodged during the contact of the coating with a second bubble, which may have facilitated coalescence.

It is unclear if the density of the particles directly influenced the properties of the bubbles. The fact that more particles remained on the surface of the coalesced bubble may partly explain the high dampening observed with PMMA particles. However, no clear correlation emerged between the maximum acceleration of the bubble surface (i.e. which is a measure of energy) and the detachment of particles. Whilst the presence of particles may give rise to a viscous (drag) resistance and alter the surface properties, the results are inconclusive in terms of how the interfacial energy balance is affected by the removing of particles (i.e. creation of new bubble surface).

The detachment of particles for the different particulate systems was compared via a modified Bond number. This number represents the balance between attachment and detachment forces. Although the magnitude of the modified Bond number was lower than theory predicted, it showed a very good consistency. It was found that the PMMA may not have followed the description of the modified Bond number theory due to its density being similar to the continuous phase. Therefore, the density of the particles appears to be an important parameter in the selection of particles to stabilise dispersed phases. In addition to the increase in the stability of the bubbles, the fact that the particles of lower density remained on the surface implies that a higher coverage is present on the coalesced bubble which may help prevent further coalescence in many bubble systems. 


\section{Supporting Information}

Details on the numerical modelling of coalescence dynamics may be found. Moreover, a video showing the lateral displacement of a portion of the particle coating may be viewed.

\section{Acknowledgements}

The Australian Research Council is acknowledged for financial support through grant DP120102305.

The authors also wish to thank Penelope Wilcher and Sin-Ying Tan for the experimental work on the PMMA particles. Kitty Tang is also acknowledged for the measurement of contact angle (galena) and sizing of particles (anatase).

\section{References}

1. Schulze, H. J.; Wahl, B.; Gottschalk, G., Determination of adhesive strength of particles within the liquid/gas interface in flotation by means of a centrifuge method. Journal of Colloid and Interface Science 1989, 128, (1), 57-65.

2. Sethumadhavan, G. N.; Nikolov, A. D.; Wasan, D. T., Film stratification in the presence of colloidal particles. Langmuir 2001, 17, (7), 2059-2062.

3. Fujii, S.; Iddon, P. D.; Ryan, A. J.; Armes, S. P., Aqueous particulate foams stabilized solely with polymer latex particles. Langmuir 2006, 22, (18), 7512-7520.

4. Hunter, T. N.; Pugh, R. J.; Franks, G. V.; Jameson, G. J., The role of particles in stabilising foams and emulsions. Advances in Colloid and Interface Science 2008, 137, (2), 57-81.

5. Binks, B. P.; Horozov, T. S., Aqueous foams stabilized solely by silica nanoparticles. Angewandte Chemie International Edition 2005, 117, (24), 3788-3791.

6. Levine, S.; Bowen, B. D.; Partridge, S. J., Stabilization of emulsions by fine particles I. Partitioning of particles between continuous phase and oil/water interface. Colloids and Surfaces 1989, 38, (2), 325-343.

7. Kaptay, G., On the equation of the maximum capillary pressure induced by solid particles to stabilize emulsions and foams and on the emulsion stability diagrams. Colloids and Surfaces A: Physicochemical and Engineering Aspects 2006, 282-283, (20), 387-401.

8. Kruglyakov, P. M.; Nushtayeva, A. V.; Vilkova, N. G., Experimental investigation of capillary pressure influence on breaking of emulsions stabilized by solid particles. Journal of Colloid and Interface Science 2004, 276, (2), 465-474.

9. Stancik, E. J.; Kouhkan, M.; Fuller, G. G., Coalescence of particle-laden fluid interfaces. Langmuir 2004, 20, (1), 90-94.

10. Bournival, G.; Ata, S.; Wanless, E. J., The roles of particles in multiphase processes: Particles on bubble surfaces. Advances in Colloid and Interface Science 2015, 225, 114-133.

11. Pugh, R. J., Foaming, foam films, antifoaming and defoaming. Advances in Colloid and Interface Science 1996, 64, 67-142.

12. Planchette, C.; Biance, A.-L.; Pitois, O.; Lorenceau, E., Coalescence of armored interface under impact. Physics of Fluids 2013, 25, (4), 1-11.

13. Ata, S., The detachment of particles from coalescing bubble pairs. Journal of Colloid and Interface Science 2009, 338, (2), 558-565.

14. Tan, S.-Y.; Ata, S.; Wanless, E. J., Direct observation of individual particle armored bubble interaction, stability, and coalescence dynamics. Journal of Physical Chemistry B 2013, 117, (28), 8579-8588. 
15. Blute, I.; Pugh, R. J.; van de Pas, J.; Callaghan, I., Silica nanoparticle sols 1. Surface chemical characterization and evaluation of the foam generation (foamability). Journal of Colloid and Interface Science 2007, 313, (2), 645-655.

16. Thompson, K. L.; Giakoumatos, E. C.; Ata, S.; Webber, G. B.; Armes, S. P.; Wanless, E. J., Direct observation of giant pickering emulsion and colloidosome droplet interaction and stability. Langmuir 2012, 28, (48), 16501-16511.

17. Ueno, K.; Hamasaki, S.; Wanless, E. J.; Nakamura, Y.; Fujii, S., Microcapsules fabricated from liquid marbles stabilized with latex particles. Languir 2014, 30, (11), 3051-3059.

18. Binks, B. P., Particles as surfactants - similarities and differences. Current Opinion in Colloid and Interface Science 2002, 7, (1-2), 21-41.

19. King, R. P., Modeling and simulation of mineral processing systems. 2nd ed.; Society for Mining, Metallurgy, and Exploration (SME): Englewood, Colorado, 2012; p 466.

20. Aktas, Z.; Cilliers, J. J.; Banford, A. W., Dynamic froth stability: Particle size, airflow rate and conditioning time effects. International Journal of Mineral Processing 2008, 87, (1-2), 65-71.

21. Johansson, G.; Pugh, R. J., The influence of particle size and hydrophobicity on the stability of mineralized froths. International Journal of Mineral Processing 1992, 34, (1-2), 1-21.

22. Trahar, W. J., A rational interpretation of the role of particle size in flotation. International Journal of Mineral Processing 1981, 8, (4), 289-327.

23. Ferin, J.; Oberdörster, G., Biological effects and toxicity assessment of titanium dioxides: anatse and rutile. American Industrial Hygiene Association Journal 1985, 46, (2), 69-72.

24. Hunter, T. N. The influence of nanoparticle \& surfactant interactions on foam stability. The University of Newcastle, Australia, 2008.

25. MacDonald, D. E.; Markovic, B.; Boskey, A. L.; Somasundaran, P., Physico-chemical properties of human plasma fibronectin binding to well characterized titanium dioxide. Colloids and Surfaces B: Biointerfaces 1998, 11, (3), 131-139.

26. Washburn, E. W., The dynamics of capillary flow. The Physical Review 1921, 17, (3), 273-283.

27. MacDonald, D. E.; Deo, N.; Markovic, B.; Stranick, M.; Somasundaran, P., Adsorption and dissolution behaviour of human plasma fibronectin on thermally and chemically modified titanium dioxide particles. Biomaterials 2002, 23, (4), 1269-1279.

28. Ata, S., Coalescence of bubbles covered by particles. Langmuir 2008, 24, (12), 6085-6091.

29. Bournival, G.; Pugh, R. J.; Ata, S., Examination of $\mathrm{NaCl}$ and $\mathrm{MIBC}$ as bubble coalescence inhibitor in relation to froth flotation. Minerals Engineering 2012, 25, (1), 47-53.

30. Bournival, G.; de Oliveira e Souza, L.; Ata, S.; Wanless, E. J., Effect of alcohol frothing agents on the coalescence of bubbles coated with hydrophobized silica particles. Chemical Engineering Science 2015, 131, 1-11.

31. Zieminski, S. A.; Whittemore, R. C., Behavior of gas bubbles in aqueous electrolyte solutions. Chemical Engineering Science 1971, 26, (4), 509-520.

32. Craig, V. S. J.; Ninham, B., W.; Pashley, R. M., The effect of electrolytes on bubble coalescence in water. Journal of Physical Chemistry 1993, 97, (39), 10192-10197.

33. Kanta, A.; Sedev, R.; Ralston, J. R., Thermally- and photoinduced changes in the water wettability of low-surface-area silica and titania. Langmuir 2005, 21, (6), 2400-2407.

34. Bournival, G.; Ata, S., Packing of particles on the surface of bubbles. Minerals Engineering 2010, 23, (2), 111-116.

35. Nowak, E.; Combes, G.; Stitt, E. H.; Pacek, A. W., A comparison of contact angle measurement techniques applied to highly porous catalyst support. Powder Technology 2013, 233, 52-64.

36. Stevens, N.; Priest, C. I.; Sedev, R.; Ralston, J. R., Wettability of photoresponsive titanium dioxide surfaces. Langmuir 2003, 19, (8), 3272-3275.

37. Miyauchi, M.; Nakajima, A.; Fujishima, A.; Hashimoto, K.; Watanabe, T., Photoinduced surface reactions on $\mathrm{TiO}_{2}$ and $\mathrm{SrTiO}_{3}$ films: Photocatalytic oxidation and photoinduced hydrophilicity. Chemistry of Materials 2000, 12, (1), 3-5.

38. Biggs, S.; Grieser, F., Atomic force microscopy imaging of thin films formed by hydrophobing reagents. Journal of Colloid and Interface Science 1994, 165, (2), 425-430.

39. Kimura, T.; Kuroda, K.; Sugahara, Y.; Kuroda, K., Esterification of the silanol groups in the mesoporous silica derived from kanemite. Journal of Porous Materials 1998, 5, 127-132. 
40. Ossenkamp, G. C.; Kemmitt, T.; Johnson, J. H., New approach to surface-alkoxylated silica with increased hydrolytic stability. Chemistry of Materials 2001, 13, 3975-3980.

41. Bournival, G.; Ata, S.; Jameson, G. J., The influence of submicron particles and salt on the recovery of coarse particles. Minerals Engineering 2014, 69, 146-153.

42. Hunter, T. N.; Wanless, E. J.; Jameson, G. J., Effect of esterically bonded agents on the monolayer structure and foamability of nano-silica. Colloids and Surfaces A: Physicochemical and Engineering Aspects 2009, 334, (1-3), 181-190.

43. Zografi, G.; Johnson, B. A., Effect of surface roughness on advancing and receding contact angles. International Journal of Pharmaceutics 1984, 22, (2-3), 159-176.

44. Smith, J.; Bournival, G.; Tan, S.-Y.; Webber, G. B.; Ata, S.; Wanless, E. J. In High speed video observations of alumina-coated air bubble coalescence, Chemeca, Perth, Australia, 28 Sept - 01 Oct, 2014; Perth, Australia, 2014.

45. Ang, Z. J.; Bournival, G.; Ata, S., Influence of frothers on the detachment of galena particles from bubbles. International Journal of Mineral Processing 2013, 121, 59-64.

46. Horvölgyi, Z.; Máté, M.; Dániel, A.; Szalma, J., Wetting behaviour of silanized glass microspheres at water-air interface: a Wilhelmy film balance study. Colloids and Surfaces A: Physicochemical and Engineering Aspects 1999, 156, (1-3), 501-508.

47. Tarimala, S.; Dai, L. L., Structure of microparticles in solid-stabilized emulsions. Langmuir 2004, 20, (9), 3492-3494.

48. $\quad$ van de Ven, T. G. M., Colloidal Hydrodynamics. Academic Press: 1989; p 582.

49. Williams, D. F.; Berg, J. C., The aggregation of colloidal particles at the air-water interface. Journal of Colloid and Interface Science 1992, 152, (1), 218-229.

50. Israelachvili, J. N., Intermolecular and surface forces. 3rd ed.; Elsevier: New York, 2011; p 674.

51. Chan, D. Y. C.; Henry Jr, J. D.; White, L. R., The interaction of colloidal particles collected at fluid interfaces. Journal of Colloid and Interface Science 1981, 79, (2), 410-418.

52. Shchipalov, Y. K., Evaluation of electrical surface properties of hydrated quartz-glass particles based on the $\zeta$-potential value. Glass and Ceramics 1999, 56, (11-12), 335-338.

53. Conley, R. F., Practical dispersion: A guide to understanding and formulating slurries. John Wiley \& Sons: 1996; p 464.

54. Schulze, H. J., Dimensionless number and approximate calculation of the upper particle size of floatability in flotation machines. International Journal of Mineral Processing 1982, 9, (4), 321-328.

55. Schulze, H. J., New theoretical and experimental investigations on stability of bubble/particle aggregates in flotation: A theory on the upper particle size of floatability. International Journal of Mineral Processing 1977, 4, (3), 241-259.

56. Goel, S.; Jameson, G. J., Detachment of particles from bubbles in an agitated vessel. Minerals Engineering 2012, 36-38, 324-330.

57. Stevenson, P.; Ata, S.; Evans, G. M., The behavior of an oscillating particle attached to a gasliquid surface. Industrial and Engineering Chemistry Research 2009, 48, (17), 8024-8029.

58. Nguyen, A. V.; Schulze, H. J., Colloidal science of flotation. Marcel Dekker, Inc.: New York, 2004; Vol. 118, p 850.

59. Miettinen, T.; Ralston, J. R.; Fornasiero, D., The limits of fine particle flotation. Minerals Engineering 2010, 23, (5), 420-437.

60. Morris, G.; Pursell, M. R.; Neethling, S. J.; Cilliers, J. J., The effect of particle hydrophobicity, separation distance and packing patterns on the stability of a thin film. Journal of Colloid and Interface Science 2008, 327, (1), 138-144.

61. Morris, G. D. M.; Neethling, S. J.; Cilliers, J. J., Predicting the failure of a thin liquid film loaded with spherical particles. Langmuir 2014, 30, (4), 995-1003.

62. Vinke, H.; Hamersma, P. J.; Fortuin, J. M. H., Particle-to-bubble adhesion in gas/liquid/solid slurries. AIChE Journal 1991, 37, (12), 1801-1809.

63. Vella, D.; Aussillous, P.; Mahadevan, L., Elasticity of an interfacial particle raft. Europhysics Letters 2004, 68, (2), 212-218.

64. Planchette, C.; Lorenceau, E.; Biance, A.-L., Surface wave on a particle raft. Soft Matter 2012, $8,2444-2451$. 
65. Mendoza, A. J.; Guzmán, E.; Martínez-Pedrero, F.; Ritacco, H.; Rubio, R. G.; Ortega, F.; Starov, V. M.; Miller, R., Particle laden fluid interfaces: Dynamics and interfacial rheology. Advances in colloid and interface science 2014, 206, 303-319.

66. Hunter, T. N.; Wanless, E. J.; Jameson, G. J.; Pugh, R. J., Non-ionic surfactant interactions with hydrophobic nanoparticles: impact on foam stability. Colloids and Surfaces A: Physicochemical and Engineering Aspects 2009, 347, (1-3), 81-89.

67. Lucassen, J., Dynamic dilational properties of composite surfaces. Colloids and Surfaces 1992, 65, (2-3), 139-149.

68. Abdel-Fattah, A. I.; El-Genk, M. S., On colloidal particle sorption onto a stagnant air-water interface. Advances in Colloid and Interface Science 1998, 78, (3), 237-266.

69. Abdel-Fattah, A. I.; El-Genk, M. S., Sorption of hydrophobic, negatively charged microspheres onto a stagnant air/water interface. Journal of Colloid and Interface Science 1998, 202, (2), 417-429.

70. Omota, F.; Dimian, A. C.; Bliek, A., Adhesion of solid particles to gas bubbles. Part 1: Modelling. Chemical Engineering Science 2006, 61, (2), 823-834.

71. Gautam, A.; Jameson, G. J., The capillary force between a bubble and cubical particle. Minerals Engineering 2012, 36-38, 291-299.

72. Chatterjee, N.; Flury, M., Effect of particle shape on capillary forces acting on particles at the air-water interface. Langmuir 2013, 29, (25), 7903-7911.

73. Chatterjee, N.; Lapin, S.; Flury, M., Capillary forces between sediment particles and an airwater interface. Environmental Science \& Technology 2012, 46, (8), 4411-4418.

74. Aramrak, S.; Flury, M.; Harsh, J. B.; Zollars, R. L.; Davis, H. P., Does colloid shape affect detachment of colloids by a moving air-water interface? Langmuir 2013, 29, (19), 5770-5780.

75. Nutt, C. W., Froth flotation: The adhesion of solid particles to flat interfaces and bubbles. Chemical Engineering Science 1960, 12, (2), 133-141.

76. Gifford, W. A.; Scriven, L. E., On the attraction of floating particles. Chemical Engineering Science 1971, 26, (3), 287-297.

77. Aveyard, R.; Clint, J. H., Particle wettability and line tension. Journal of the Chemical Society, Faraday Transactions 1996, 92, (1), 85-89.

78. Pitois, O.; Chateau, X., Small particle at a fluid interface: Effect of contact angle hysteresis on force and work of detachment. Langmuir 2002, 18, (25), 9751-9756.

79. Ralston, J.; Fornasiero, D.; Hayes, R., Bubble-particle attachment and detachment in flotation. International Journal of Mineral Processing 1999, 56, (1-4), 133-164.

80. Stover, R. L.; Tobias, C. W.; Denn, M. M., Bubble coalescence dynamics. AIChE Journal 1997, 43, (10), 2385-2392.

81. Bournival, G.; Ata, S.; Karakashev, S. I.; Jameson, G. J., An investigation of bubble coalescence and post-rupture oscillation in non-ionic surfactant solutions using high-speed cinematography. Journal of Colloid and Interface Science 2014, 414, 50-58.

82. Cheng, T.-W.; Holtham, P. N., The particle detachment process in flotation. Minerals Engineering 1995, 8, (8), 883-891.

83. Denkov, N. D.; Ivanov, I. B.; Kralchevsky, P. A.; Wasan, D. T., A possible mechanism of stabilization of emulsions by solid particles. Journal of Colloid and Interface Science 1992, 150, (2), 589-593.

84. Nguyen, A. V.; Ralston, J.; Schulze, H. J., On modelling of bubble-particle attachment probability in flotation. International Journal of Mineral Processing 1998, 53, (4), 225-249.

85. Jameson, G. J., New directions in flotation machine design. Minerals Engineering 2010, 23, (11-13), 835-841. 